\title{
Die Struktur einiger Kristalle, gedeutet nach ihrer Beugung von Röntgen-Strahlen.
}

\author{
Von \\ W. L. BragG. ${ }^{1}$ \\ Mit 14 Figuren im Text und 1 Tafel.
}

Ein neues Verfahren zur Untersuchung der Struktur eines Kristalles ist aufgefunden worden durch die Arbeit von $L_{A U E}{ }^{2}$ und seinen Mitarbeitern über die Beugung von $X$-Strablen durch Kristalle. Die Erscheinungen, welche sie als Erste untersuchten, und die seitdem von vielen anderen beobachtet worden sind, lassen sich ungezwungen in der von LAUE vorgeschlagenen Weise erklären. Dieser nahm an, daB elektromagnetische Wellen von sehr kurzer Wellenlänge Beugung erfahren durch eine Reihe sehr kleiner Hindernisse, die in einem regelmäBigen Punktsystem im Raum angeordnet sind. Bei der Analyse des mit einem Zinkblendekristall erhaltenen Interferenzmusters kam LaUE in seiner ursprünglichen Mitteilung zu dem Schluß, daß die primäre Strahlung ein aus schmalen Banden bestehendes Spektrum besäBe, und sich in der Tat aus einer Reihe von 6 oder 7 angenähert homogenen Wellenzügen zusammensetze.

In einer neueren Mitteilung versuchte ich zu zeigen, daB die Notwendigkeit zur Annahme solch' komplizierter Verhältnisse sich vermeiden lasse, wenn man für den kubischen Kristall der Zinkblende ein Punktsystem voraussetzt, das von dem von LaUE benutzten abweicht. Ich nahm an, daB die beugenden Zentren in einem einfachen kubischen Raumgitter angeordnet seien, und daB das Element des Musters ein Würfel sei mit einem Punkt in jeder Ecke und einem im Mittelpunkt jeder Würfelfläche. Hierdurch wurde eine einfachere Auffassung der Strahlung ermöglicht. Sie konnte über ein weites Gebiet von Wellenlängen als kontinuierlich angesehen werden oder als eine Reihe von unabhängigen Impulsen,

${ }^{1}$ Aus Proc. Roy. Soc. A. 89 (1913), 248 ins Deutsche übertragen von I. KopPEL-Berlin.

2W. Friedrich, P. Knspping u. M. Lade, Mïnch. Akad. Ber., Jani 1912.

3 Proc. Cambr. Phil. Soc. November 1912.

Z. anorg. Chem. Bd. 90. 
und es bestand weiterhin keine Notwendigkeit, die Existenz von Linien oder schmalen Banden in ihrem Spektrum anzunehmen.

In dieser Mitteilung soll die für die Zinkblende benutzte Art der Analyse weiter auf einige andere Kristalle ausgedehnt werden, insbesondere auf die einfachen Alkalibalogenide.

Bei der Behandlung der Beugung von Wellen durch ein Raumgitter, wie es ein Kristall vorstellt, ist der Fall der einfachste, bei dem die Beugung verursacht wird durch eine Reihe von Punkten, die in einem Raumgitter, nach Art der 14 von BRavaIs aufgestellten, angeordnet sind. In diesem Fall ist jeder Punkt identisch mit jedem anderen Punkt der Anordnung, und es ist immer möglich, ein Element des Musters aufzufinden, das aus einem Parallelepiped mit einem Punkt in jeder Ecke besteht: dann werden in jedem Raum ebenso viele Parallelepipede vorbanden sein wie Atome. Die Punkte können bezogen werden auf drei Achsen, parallel den Kanten des Parallelepipedes, und wenn man einen der Punkte als Ursprung wäblt, so können die Koordinaten der anderen geschrieben werden

$$
x= \pm p a \quad y= \pm q b \quad s= \pm r e,
$$

wo $p, q, r$, ganze Zahlen sind, und $a, b, c$ den Seiten des Parallelepipedes entsprechen, somit den Achsenverhältnissen des Raumgitters proportional sind. Werden die Achsen in dieser Weise gewählt, so werden die Koordinaten der Punkte, sowie die Gleichungen, der durch eine gegebene Auswahl von Punkten hindurchgehenden Ebenen in der möglichst einfachen Weise ausgedrückt.

Eine Reihe von Impulsen falle auf dies Raumgitter, und dio Fortpflanzungsrichtung habe eine gegebene Beziehung zu den Achsen des Systems. Da jeder Impuls über jeden Punkt fortschreitet, breitet sich von diesem eine Beugungswelle aus, und es wird gezeigt werden, daB die Beugungswellen von allen Punkten, die durch einen einfallenden Impuls hervorgerufen sind, sich in gewissen Richtungen vereinigen unter Bildung von Wellenzügen, die das Fleckenmuster erzeugen, das in Lades Beugungsmustern auftritt. Dies kann in der folgenden Weise geschehen:

Wenn das Achsenverhältnis des Raumgitters mit $a, b, c$ bezeichnet wird, so ist jede Ebene, welche die Abschnitte $p a, q b$, re auf den Achsen liefert ( $p, q, r$ sind ganze Zahlen), parallel einer ganzen Schar von Ebenen, auf denen man die Punkte des Systems angeordnet denken kann. Ebenen, wie diese, bilden die Flächen eines Kristalles. Wenn ein Impuls auf eine Anzahl Punkte einer 
Ebene fällt, vereinigen sich die Wellen von diesen Punkten zu einer Wellenfront, die von der Ebene regelmäBig reflektiert erscheint. Da eine Schar von Ebenen, eine hinter der anderen, in regelmäBigen

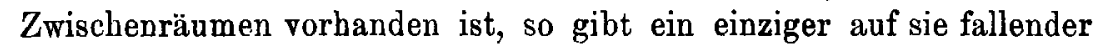
Impuls Veranlassung zu einem reflektierten Wellenzug.

Wenn demnach ein schmales Bündel $X$-Strahlen auf einen Kristallschnitt fällt, so wird ein Teil der Energie ohne Ablenkung durchgelassen, ein Teil wird aber auch reflektiert von den Kristallebenen, die potenziell im Kristall vorhanden sind. Die Reibe der durch Reflexion in dieser Weise entstehenden engen Bündel ist es, die das Muster von Flecken entstehen lassen, das in der Photographie erscheint.

Jeder Schar paralleler Ebenen können ganzzablige Indizes $(h, k, l)$ zuerteilt werden, wie man auch die Flächen eines Kristalles benennt, und ein Fleck kann darnach eingeordnet werden als reflektiert von der $(h, k, l)$.Schar von Ebenen. Es sind bier die ganzen Zahlen $(h, k, l)$ die reziproken Werte der Längen, die eine Parallelebene auf den Bezugsachsen abschneidet. Es sind die Miluerschen Indizes und die Gleichung der Ebene ist:

$$
h \frac{x}{a}+k \frac{y}{b}+l \frac{z}{c}=\text { Ganze Zahl. }
$$

Sie können betrachtet werden als die Parameter eines jeden Fleckes im Muster, und sie sind genau den von LAUE bei seiner ursprünglichen Behandlung dieses Gegenstandes benutzten Parametern $\left(h_{1}, h_{2}, h_{3}\right)$ gleichwertig. LuUE definierte seine Parameter, indem er sagte, daB die von einem Punkt im Ursprung der Koordinaten gebeugte Welle um $h_{1}, h_{2}, h_{3}$ Wellenlängen in den betrachteten Richtungen hinter den Wellen zurückbleibt, die von ihren Nachbaren auf den $x-, y$-, $z$-Achsen ausgehen. Demnach sind die Wellen von allen Atomen in der Ebene

$$
h_{1} \frac{x}{a}+h_{2} \frac{y}{b}+h_{3} \frac{z}{c}=0
$$

in Phase mit der vom Ursprung ausgehenden, und im allgemeinen sind die Wellen von allen Punkten der Ebene

$$
h_{1} \frac{x}{a}+h_{2} \frac{y}{b}+h_{3} \frac{z}{c}=\text { Ganze Zahl }
$$

in derselben Phase. Wir werden zu demselben SchluB geführt wie vorher, daB die Richtung der abgebeugten Wellenfront dieselbe ist, 
in welcher das primäre Bündel durch Ebenen reflektiert wird, deren Millersche Indizes $h_{1}, h_{2}, h_{3}$ sind. Wie aber jetzt deutlich wird, ist es wichtig, die Tatsache $\mathrm{zu}$ beachten, daB die Kristallstruktur allein die genaue Lage der Interferenzmaxima festlegt, ganz unabhängig von der Existenz homogener Komponenten bestimmter Wellenlänge in der einfallenden Strahlung, und deswegen ist eine Behandlungsweise gewählt worden, bei der jede Bezunahme auf die Wellenlänge vermieden worden ist.

Ein Fleck kann in jeder Lage erscheinen, die der Retiexion an irgend einer Schar von Ebenen mit ganzzahligen Indizes $(h k l)$ entspricht. Bei der Erzeugung eines Musters durch Auftreffen gebeugter X-Strahlen auf eine photographische Platte, kann nur eine Auswahl von Ebenen wirksam sein, da sich nicht eine unbegrenzte Anzahl von Flecken vorfindet. Diese Flecke, die das Muster bilden, haben sehr verschiedene Intensitäten, und man kann niemals sagen, da $B$ sie vollständig wären, sondern nur, daB in gewissen Fällen die Strahlen zu schwach sind, um eine Einwirkung auszuüben. Es scheint jedoch, daB durch Gruppierung der Ebenen, welche die wichtigsten Flecke eines Musters reflektieren, ein Schlüssel erhalten werden kann für die wahre Anordnung des Punktsystemes der beugenden Zentren. Das Punktsystem, das die einfachste Deutung des Musters gestattet, muB als Darstellung der Kristallstruktur angenommen werden.

Wenn eine Photographie aufgenommen ist an einem Kristall, so wird eine Analyse derselben notwendig, um einem jeden Fleck des Musters die richtigen Parameter $h, k, l$, also die Miluenschen Indices der Fläche, von der der Fleck reflektiert ist, zuzuerteilen. Hat man sich über die Bezugsachsen des Kristalles entschieden, so wird das folgende Verfahren anwendbar befunden werden, einerlei, welches ihre Neigungen zueinander und die Achsenverbältnisse sein mögen.

Die wichtigeren Ebenen des Systemes sind die dicht mit Punkten bepackten, und hieraus folgt, daB diese Ebenen Reihen enthalten, nach welchen die Punkte sich in enger Lagerung befinden. Dies sind die wichtigen „Punktreihen" des Kristalles, und jede dieser Punktreihen hat eine Schar mit ihr paralleler wichtiger Ebenen. Die drei Achsen selbst sind die hervortretensten Beispiele dieser Punktreihen. Die "Zonenachse" von Ebenen, die zu einer gemeinsamen Zone gehören, ist gleichfalls eine solche Richtung. Es besteht eine einfache Beziehung zwischen den Punkten, die in 
Ebenen ein und derselben Zone reflektiert werden. Das reflektierte Bündel liegt immer auf einem Kreiskegel mit dem Scheitel im Kristallschnitt, der Zonenachse als Achse und der Richtung des primären Strahles als einer Erzengenden. Dieser Kegel schneidet die photographische Platte in einer Ellipse, die durch den Mittelpunkt des Musters geht, und alle in Ebenen dieser Zone reflektierten Flecke liegen auf dieser Ellipse. Die Anordnung der Flecke auf Ellipsen ist in den Interferenzmustern sehr deutlich (s. Fig. 11), und die Ellipse kann unmittelbar gezogen werden. Eine kleine Rechnung zeigt, zu welcher Zonenachse jede Ellipse gehört, und indem man einen gegebenen Fleck als Schnittpunkt zweier Ellipsen bezeichnet, wird die Berechnung der Indizes $h, k, l$ dieses Fleckes ermöglicht. Für

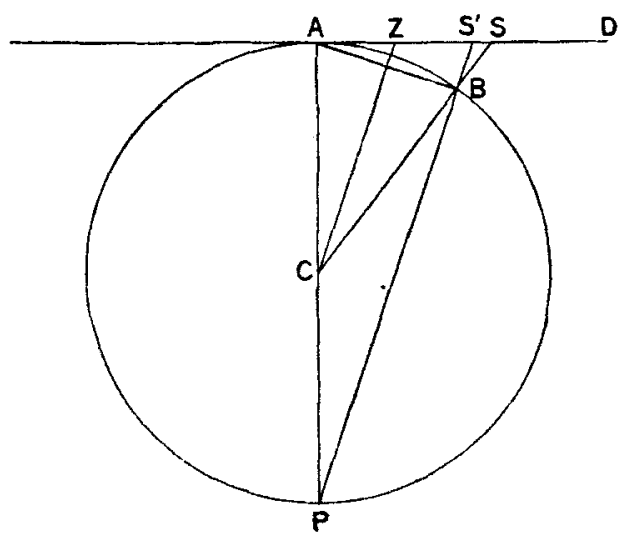

Fig. 1. jede Zone von Ebenen haben wir die Beziehung $h U+k V+l W=0$, wo $(U, V ; W)$ das Zonensymbol ist, d. h. die Schar der Richtungsverhältnisse der Zonenachse.

Wenn man zeichnerisch ein Interferenzmuster darstellt, ist es sehr unbequem, die Ellipsen zu ziehen, in deren Schnittpunkten die Flecke des Musters liegen. Es ist einfacher, eine Erweiterung der gewöhnlichen stereographischen Projektion der Kristallographie zu benutzen. Die Fig. 1 wird dies klar machen. Der Schnitt des Kristalles sei bei $C$ aufgestellt, dem Mittelpunkt der Kugel, die in der Figur durch den Kreis $A B P$ dargestellt wird; die Richtang des einfallenden Strahles sei von $P$ nach $C$. Die Strahlen, die den Kristall unabgelenkt durchdringen, fallen auf die photographische Platte $A D$ bei $A$. Es sei $C Z$ die Richtung einer Zonenachse. Die in Ebenen dieser Zone reflektierten Bündel liegen auf einem Kreiskegel mit dem Scheitel bei $C$, der Achse $C Z$ und den beiden Erzeugenden $C A$ und $C B$.

Dieser Kegel schneidet die Kugel in einem Kreis, dessen Durchmesser $A B$ ist, und nach der bekannten Eigenschaft der stereo- 
graphischen Projektion ist die Projektion dieses Kreises auf die Ebene $A D$ von Pol $P$ gleichfalls ein Kreis. Der Mittelpunkt dieses Kreises liegt bei $Z$, da $A Z=Z S$, und $Z$ der Punkt ist, wo die Zonenachse die photographische Platte trifft.

Das Muster der Flecke sei durch die gebeugten Bündel auf der Kugel $A B P$ erzeugt, und dieses Muster werde auf die Ebene $A D$ von Pol $P$ aus projiziert. Die Elecke, die durch Reflexion in Ebenen einer Zone erzeugt sind, liegen jetzt auf einem Kreise, der seinen Mittelpunkt dort hat, wo die Zonenachse die Platte $A D$ schneidet. Der Fleck bei $S$, der durch das reflektierte Bündel $C B$ erzeugt wird, stellt den Fleck bei $S$ im transformierten Muster dar. Die Verzerrung des Fleckenmusters durch die Transformation ist sehr gering, ausgenommen in den vom Mittelpunkt entfernten Gegenden, und Kreise sind viel leichter zu ziehen als Ellipsen. Dies ist bei allen Zeichnungen in dieser Mitteilung durchgeführt worden. Bei der Konstruktion einer Zeichnung wurde ein Punkt gewählt, in welchem die einfallenden Strahlen die photographische Platte treffen Dies ist $A$ in der Figur. Dann fand man solche Punkte wie $Z$, wo die wichtigsten Zonenachsen die Platte treffen, durch Rechnung, welche leicht ist, wenn der Kristall symmetrisch zu dem Bündel der X-Strahlen liegt. Es wird dann ein Kreis gezeichnet mit dem Mittelpunkt $Z$ und dem Radius $Z A$. Wenn dies für jede Zonenachse ausgeführt ist, liefern die Schnittpunkte der Kreise die stereographischen Projektionen der reflektierten Flecke.

Wir wählen als Beispiel für die Anwendung dieser Analyse den sehr einfachen Fall des Kaliumchlorids. Das Beugungsmuster, das erhalten wird, wenn X-Strahlen normal auf eine parallel zur Würfelfläche (001) geschnittene Platte fallen, wird in Fig. 2 der Tafel II dargestellt und seine stereographische Projektion in Fig. 3, die auch die Indizes der reflektierenden Ebenen enthält, welche den verschiedenen Punkten der Photographie entsprechen.

Kaliumchlorid ist kubisch, und die angegebenen Indizes für die Ebenen sind diejenigen, welche man erhält, wenn man die Kanten des Würfels als Bezugsachsen wählt.

Die Kreise entsprechen Zonenachsen, für die

$$
\left.\begin{array}{ll}
U=0 & V= \pm 1 \\
U= \pm 1 & V=\quad 0
\end{array}\right\} W=0,1,2,3,4,5,
$$

und ihre Schnittpunkte geben die Flecke, die in allen Ebenen der 
Form $(h k 1)$ reflektiert werden, wo $h$ und $k$ zwei beliebigen Zahlen der folgenden Reihe ostsprechen.

$$
0, \pm 1, \pm 2, \pm 3, \pm 4, \pm \tilde{0}
$$

In der Zeichnung des Kaliumchloridmusters sind die Flecke durch Punkte dargestellt, deren Größe die Stärke des entsprechenden Fleckes in der Photographie anzeigt.

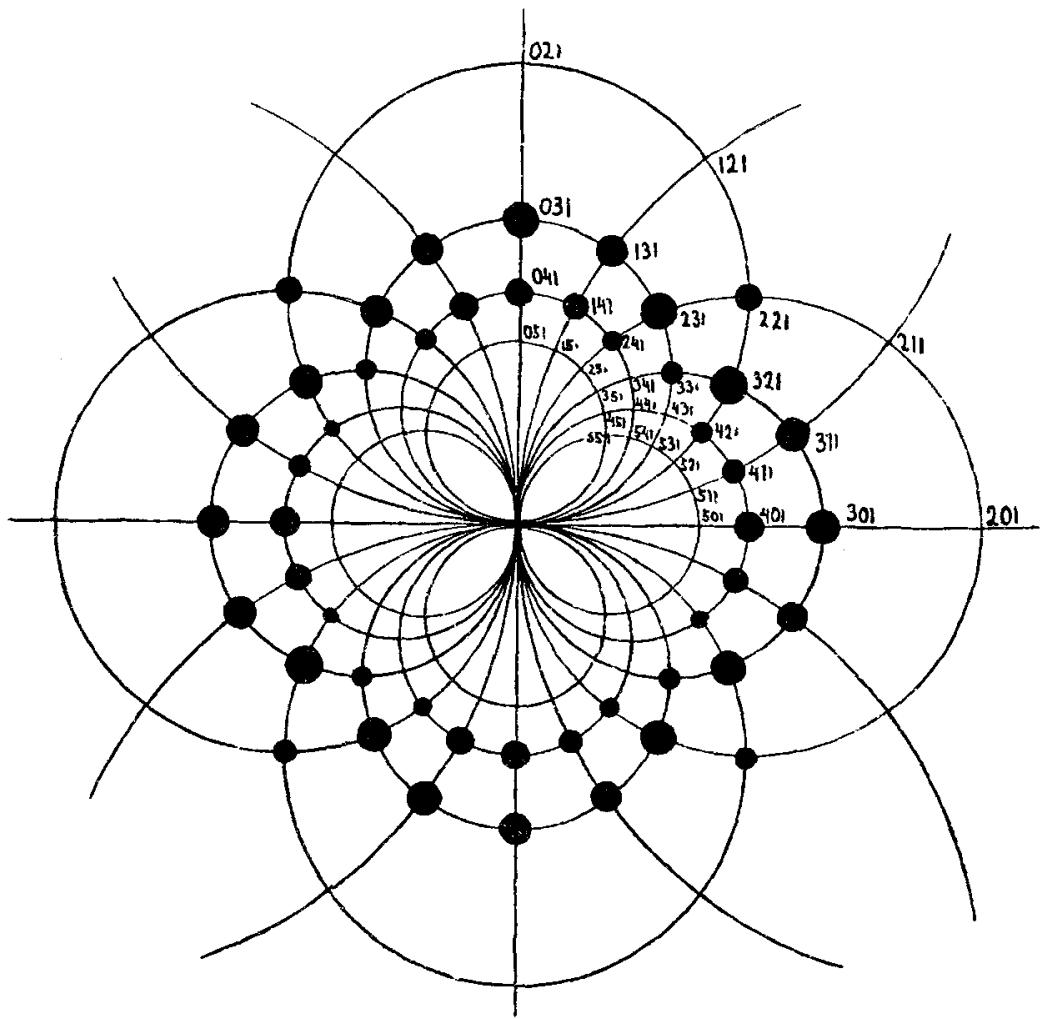

Fig. 3. Kaliumehlorid.

Man erkennt, wie vollständig das Muster ist, und wie in einem gewissen Gebiet jeder Schnittpunkt durch einen Fleck der Photographie dargestellt wird.

Dies kann auch in anderer Weise gezeigt werden. Unten steht eine Tabelle der Werte von $h$ und $k$, während $l$ immer gleich 1 angenommen ist In dem Quadrat, das gegebenen Werten von $h$ und $k$ entspricht, ist ein Zeichen gesetzt, das die Größe des entsprechenden Fleckes andeuten soll, während den leergelassenen Quadraten kein Fleck entspricht. 
Tabelle 1. $\mathrm{KCl}$.

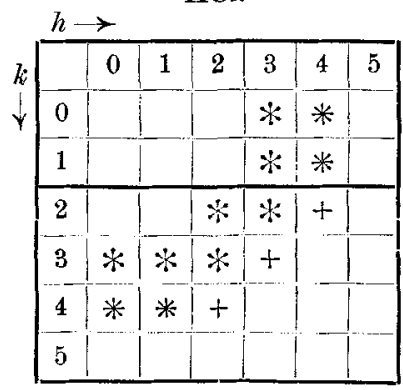

Diese Tabelle enthält ein Verzeichnis aller in der Photographie erscheinenden Flecke. Sie deutet darauf, daB die Beugung durch ein einfaches würfelförmiges Raumgitter bedingt ist, denn wenn das elementare Parallelepiped ein Würfel ist, dessen Kanten als Achsen gewählt werden, so nehmen die Indizes der Ebenen natürlicherweise diese einfache Form an.

Es möge eine Reihe von Ebenen angenommen werden, für die zwei Indizes konstant sind, während der dritte sich ändert z. B. die Reihe 031, 131, 231, 331, 431 usw. Diese Ebenen werden sich für alle Eigenschaften, wie Netzdichte, gegenseitige Entfernung usw. dann, aber auch nur dann, in dieser Reihenfolge anordnen lassen, wenn die Indizes auf Achsen parallel zu den Seiten des elementaren Parallelepipedes bezogen sind. Ist diese Bedingung befriedigt, dann ändern sich alle Eigenschaften der Ebenen in geordneter Weise, wenn man durch die Reihen fortgeht. Die reflektierende Kraft der X-Strahlen ist eine besondere Eigenschaft der Ebenen, die, wie man aus den Photographien sieht, sich kontinuierlich für jede solche Reihe, wie sie oben angeführt wurde, ändert, und deswegen ist das beugende System ein Raumgitter mit rechtwinkelig gestellten Achsen und einem Würfel als Elementarparallelepiped.

Hier kann eingewandt werden, daB es verständlich wäre, wenn eine komplizierte Struktur, sowie eine aus homogenen Kompenenten bestehende Strahlung gerade im Falle des Kaliumchlorides zufälligerweise ein täuschend einfaches Muster gibt. DaB dies nicht der Fall ist, läbt sich erkennen, wenn man den Kristall aus seiner symme. trischen Lage verschiebt, worauf man dann ein gestörtes Interferenzmuster erhält. Es ist noch so ausgeprägt wie vorher, aber natürlich nicht mehr symmetrisch. Entsprechende Flecke der beiden Muster werden nun durch verschiedene Wellenlängen erzeugt, und es ist 
klar, dab nun nichts mehr von der Natur der homogenen Komponenten der einfallenden Strahlung vorhanden sein kann. Der Gegensatz dieses Musters mit den für Kaliumbromid, Kaliumjodid, Steinsalz und Zinkblende charakteristischen, die alsbald besprochen werden sollen, wird dies klarer hervortreten lassen.

Alle sehr intensiven Flecke der Photographie liegen ungefähr in demselben Abstand vom Mittelpunkt des Musters. Ein Kreis kann so gezogen werden, daB die ihm benachbarten Flecke sehr stark sind, während die weiter entfernten schwächer, und die sehr weit entfernten so schwach sind, daB sie nicht erscheinen. Alle Schnittpunkte von Ellipsen, die in der Nähe dieses Kreises liegen, werden durch einen Fleck dargestellt. Diejenigen Ebenen reflektieren sehr stark, für die der Spiegelungswinkel zwischen 12 und $2^{\circ}$ liegt, und um eine Erklärung hierfür zu finden ist es notwendig, die Frage nach der "Wellenlänge" der gebeugten Strahlen zu erörtern. Dies soll nun geschehen.

Wenn ein Impuls anf eine Schar von Ebenen fällt, die in regelmäBigen Zwischenräumen hintereinander liegen, so wird er als Wellenzug reflektiert, und für Wellen die einen Fleck hervorrufen, muß man Wellenlängen annehmen, die gegeben sind durch die Gleichung

$$
n \lambda=2 d \sin \Theta
$$

wo $\Theta=$ Spiegelungswinkel, $d=$ Abstand zwischen den aufeinanderfolgenden Ebenen und $n$ eine ganze Zahl, etwa 1, 2 oder 3 usw. ist. Ein anderer Weg, diese Erscheinung zu betrachten, besteht darin, daB man annimmt, die einfallende Strahlung sei zusammengesetzt aus homogenen Wellenzügen von allen Wellenlängen mit einer charakteristischen Verteilung der Energie in ihrem Spektrum, wobei man dann voraussetzen muB, daB die parallelen Ebenen nur den Teil des Spektrums reflektieren, für den die Beziehung $n \lambda=2 d \sin \Theta$ zutrifft. In dem hier behandelten Fall eines kubischen Raumgitters wird die Beziehung die Form erhalten

$$
n \lambda=\frac{2 a}{l} \sin ^{2} \theta
$$

wo $a$ der Abstand zwischen benachbarten Punkten in der Richtung der kubischen Achsen ist, denn eine einfache Rechnung zeigt, daß $d=a \sin \Theta / l$. Da $l$ gleich der Einheit für alle Flecke der Kaliumchloridphotographie ist, entsprechen Flecke mit demselben $\Theta$, die in derselben Entfernung rom Mittelpunkt des Musters liegen, derselben Wellenlänge. 
Der Ring von intensiven Flecken in dem Muster deutet auf starke Reflexion eines gewissen Teiles des Spektrums der einfallenden Strablen. Beide Bedingungen sind besonders günstig ${ }^{1}$ für die Reflexion dieser Wellenlänge oder die einfallenden Strahlen haben eine große Menge ihrer Energie in diesem Teil des Spektrums.

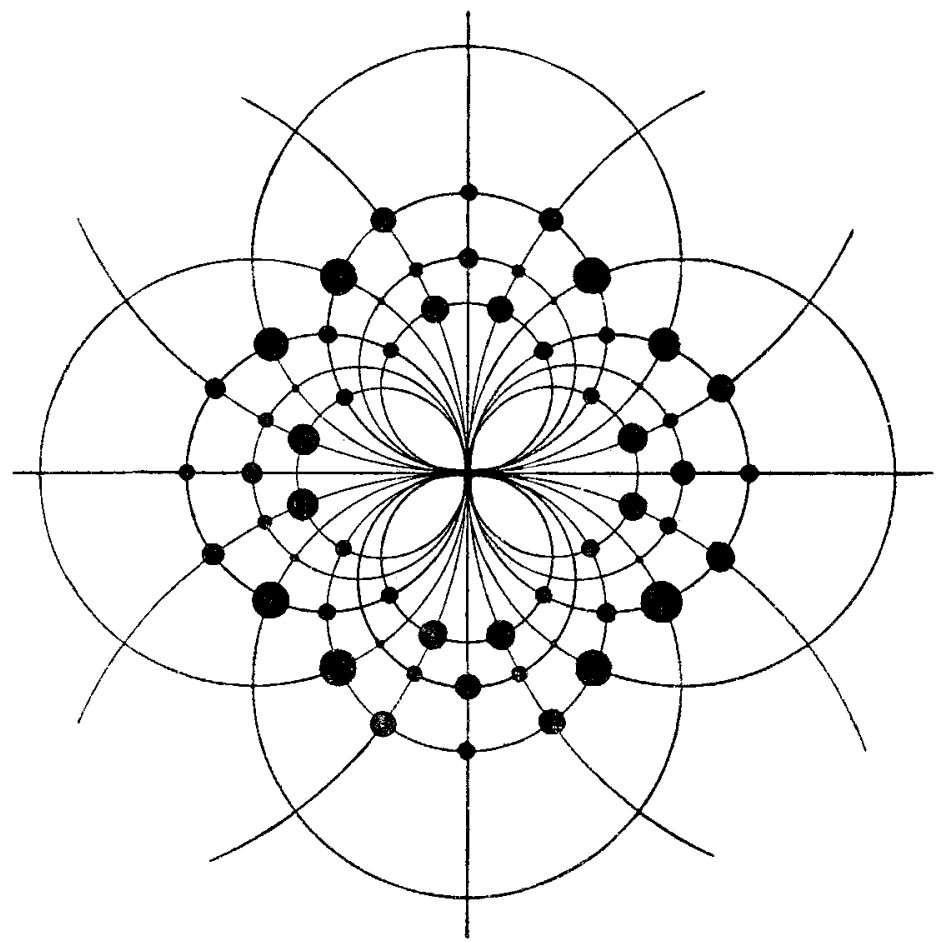

Fig. 4. Kaliumbromid.

Es ist von Interesse, das einfache Muster des Kaliumchlorides mit denen von Kaliumjodid, Kaliumbromid, Flußspat und Zinkblende zu vergleichen.

Die stereographische Projektion von $K B r(100)$ ist in Fig. 4 dargestellt, die von $K J$ ist sehr ähnlich. Diese beiden gleichen den Mustern von Zinkblende und FluBspath; sie stehen im ausgesprochenen Gegensatz zu dem von Kaliumchlorid.

Es ist augenscheinlich, daß nunmehr ein Faktor eingetreten ist, der die einfache Anordnung der Flecke, welche für Kaliumchlorid charakteristisch ist, zerstört. Die Flecke erscheinen jetzt nicht mehr bei jedem Schnittpunkt der Ellipsen innerhalb einer gewissen Gegend

\footnotetext{
${ }^{1}$ Siehe die Tabelle 2 und 3.
} 
wie in Fig. 3. Eine Aufstellung der Intensitäten der Flecke von Zinkblende und Kaliumbromid wird dies klar machen. ${ }^{1}$

Tabelle 2.

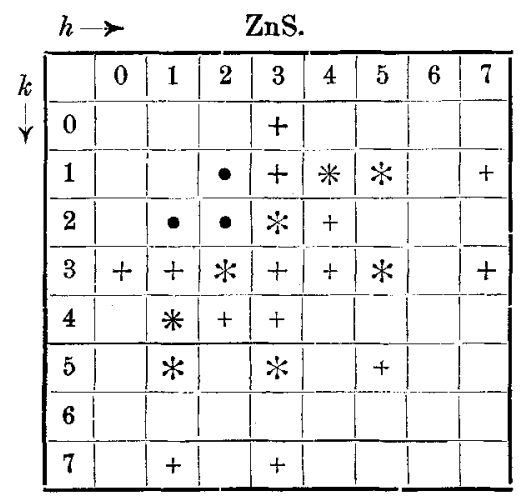

Tabelle 3.

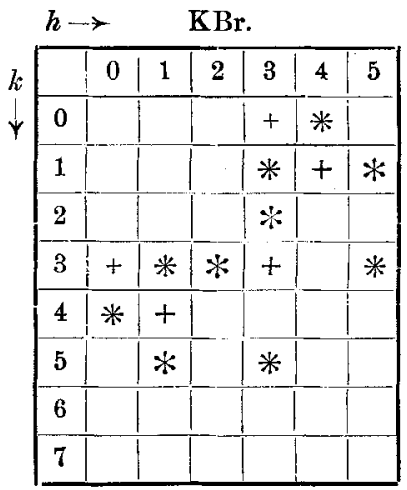

Es sind auch bei der Zinkblende verschiedene Flecke, für welche der Wert 3 für $l$ gewählt werden muB, wenn $h$ und $k$ ganze Zahlen sein sollen, allerdings nicht viele. Die Betrachtung der Tabelle 2 führte in der früheren Mitteilung ${ }^{2}$ zu dem Schlub, daB die beugenden Zentren des Zinkblendekristalles im kubischen Raumgitter mit Punkten inmitten der Würfelfächen angeordnet seien.

Eine Prüfung der Ebenen, in denen Reflexion stattfindet, zeigt, daß ein Unterschied auftritt, zwischen denen, deren Indizes alle ungerade und jenen, die einen oder mehrere gerade Indizes besitzen. Berücksichtigt man allein die Ebenen mit ungeraden Indizes, so ist das Schema so vollständig, wie für die Flecke des Kaliumchloridkristalles. (Siehe Tabelle 4).

Das Gleiche gilt für die Ebenen mit geraden Indizes, ${ }^{3}$ abgesehen davon, daB die starken Flecke alle vom Mittelpunkt des Bildes weiter entfernt sind.

${ }^{1}$ Der wichtigste Faktor in dieser Beziehung ist wahrscheinlich die Dicke des benutzten Kristallschnittes. Wie später gezeigt werden wird, begiinstigt die Benutzung eines dünnen Kristallschnittes die Reflexion der längeren Wellenlängen und die entsprechenden Flecke kommen stark zum Vorschein, während bei einem dicken Kristallschnitt die Reflexion der kürzeren bevorzngt ist.

2 Proe. Cambr. Phil. Soc. November 1912.

3 Der Fleck 041 bildet eine Ausnahme, in der Tat fehlt es nicht an Gründen, daB die Annahme einer Beugung durch ein Raumgitter mit Flächenmittelpunkten dies Muster nicht völlig erklärt. Der Grund hierfür wird klar werden, wenn die Rolle von verschiedenartigen Atomen bei der Beugung besprochen wird. 
Tabelle 4.

$\mathrm{ZnS}$ (nur ungerade Indizes).

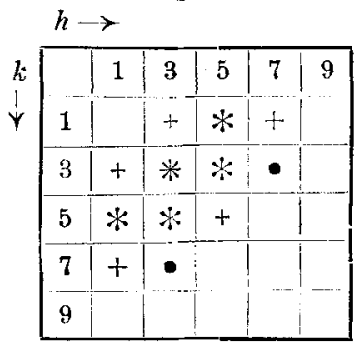

Ungerade Ebenen.
Tabelle 5.

$\mathrm{ZnS}$ (gerade Indizes).

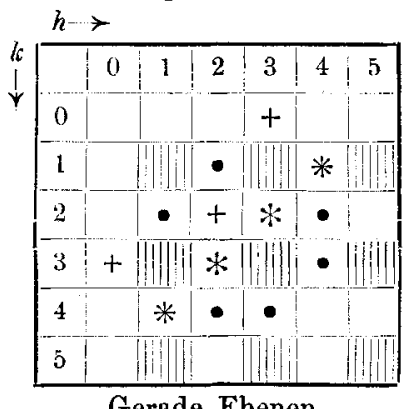

Gerade Ebenen.

Dieser Unterschied läBt sich erklären, ohne daB wir gezwungen wären anzunehmen, das beugende System sei etwas anderes als ein einfaches System identischer Punkte. Es genügt dazu die Annahme, daB das Punktsystem auch in den Mittelpunkten der Würfellächen Punkte besitzt, ebenso wie in den Würfelecken. Man nehme ein kubisches Punktsystem der ersten Art, das nur Punkte an den Würfelecken besitzt und füge noch Punkte in den Mitten der Würfelflächen ein, um es in ein Punktsystem „dritter Art" überzuführen. (Das Punktsystem ,zweiter Art" ist der Würfel mit Mittelpunkt). Die Abstände der Ebenen, die ungerade Indizes $(h, k, l)$ besitzen, sind nicht geändert durch die Einfügung der neuen Punkte, denn diese liegen alle in den ursprünglichen Ebenen und vermehren nur die Dichtigkeit der Punkte. Andererseits liegen für die Ebenen mit geradem Index einige der neuen Punkte auf halbem Wege zwischen den ursprünglichen Ebenen, und der Abstand der aufeinanderfolgenden Ebenen dieser Art muB nun halbiert werden; und dies muB auch mit den Wellenlängen der reflektierten Bündel geschehen.

Die Deutung des Zinkblendemusters ist nunmehr einfach. Wir haben gesehen, dab nur die Ebenen mit ungeraden Indizes eine rollständige Gruppe bilden. Es finden sich weniger Flecke, die der Reflexion in Ebenen mit einem geraden Index entsprechen; denn diese Ebenen sind im Verhältnis zu jenen weniger dicht besetzt und von komplizierterer Natur. Überdies sind sie gleichzeitig weniger weit getrennt, und demnach liegen die intensiven Flecke mit geraden Indizes weiter vom Mittelpunkt ab; der Einfallswinkel muB vergrößert werden, damit diese Ebenen die Region des Spektrums, die starke Flecke liefert, reflektieren. Durch Annahme des dritten 
kubischen Raumgitters an Stelle des ersten entsprechen wieder alle intensiven Flecke des Musters derselben Wellenlängenregion.

Der Unterschied zwischen der Beugung durch die beiden kubischen Raumgitter läBt sich klarer erkennen bei der Analyse eines Musters von dreifacher Symmetrie, das man erhält, wenn der eintretende Strahl senkrecht auf eine Platte fällt die senkrecht zu einer dreizähligen Achse geschnitten ist. Betrachtet man die Punkte des Raumgitters von diesem Gesichtspunkt, so sind sie besondere Fälle des trigonalen rhomboedrischen Raumgitters, das zu den Typen von Bravais gehört.

Die Achsen, auf welche die Flecke bezogen werden müssen, sind für die beiden kubischen Gitter nicht dieselben. Wenn die Punkte allein in den Ecken des Würfels liegen, so sind die Achsen, nach denen sie am meisten benachbart sind, die Würfelkanten, und der Würfel selbst ist das elementare Parallelepiped. Wenn aber auch Punkte in den Mitten der Würfelfächen vorhanden sind, so bilden drei Halbdiagonalen der Würfelflächen, die sich in einer Ecke treffen, drei Kanten des elementaren Parallelepipeds. Im ersten Falle sind die Winkel zwischen den Achsen rechte Winkel im zweiten Falle sind es Winkel ron $60^{\circ}$.

Es wird nunmehr klar sein, daB bei der Konstruktion des stereographischen Diagrammes, das die Lagen der von den einfachen Ebenen eines trigonal-rhomboedrischen Raumgitters reflektierten Flecke liefert, ein solches Diagramm die Muster aller rhomboedrischen Gitter darstellt, da dio Änderung des Winkels zwischen den Achsen d. b. des rhomboedrischen Winkels nur eine Änderung im Maßstabe des Diagramms bedingt. Der Radius der Kugel, den man bei der Projektion benutzt, wird natürlich immer von gleicher GröBe angenommen.

Wenn die Punkte, wo die drei Achsen des Gitters das Diagramm schneiden, gegeben sind, können die entsprechenden Punkte für die anderen Zonen unmittelbar gefunden und das ganze Diagramm gezeichnet werden. Eine derartige Zeichnung ist in Fig. 5 dargestellt. $X Y Z$ sind die Achsenpunkte. Nun hat unsere Untersuchang des Musters der vierzähligen Symmetrie beim Kaliumchlorid und der Zinkblende gezeigt, daB die Achsen des Gitters, das von diesem Standpunkt ein besonderer Fall des rhomboedrischen Gitters ist, im ersten Fall Winkel von $90^{\circ}$ und im zweiten Fall Winkel von $60^{\circ}$ bilden. Demnach sollte es möglich sein, das dreizählige Muster von Kaliumchlorid und Zinkblende auf dasselbe trigonale Gitterdiagramm 
zu beziehen, wobei jedoch der MaBstab in beiden Fällen verschieden ist. Dieser SchlnB wird durch Versuch durchaus bestätigt. Das von Zinkblende gelieferte Muster wurde von $L_{A U E}$ in seiner ursprünglichen Arbeit veröffentlicht. Die stereographische Projektion des Zinkblende- und Kaliumchloridmusters sind in den Fig. 6 und 7 dargestellt. Durch diesen Vergleich soll gezeigt werden, daB die

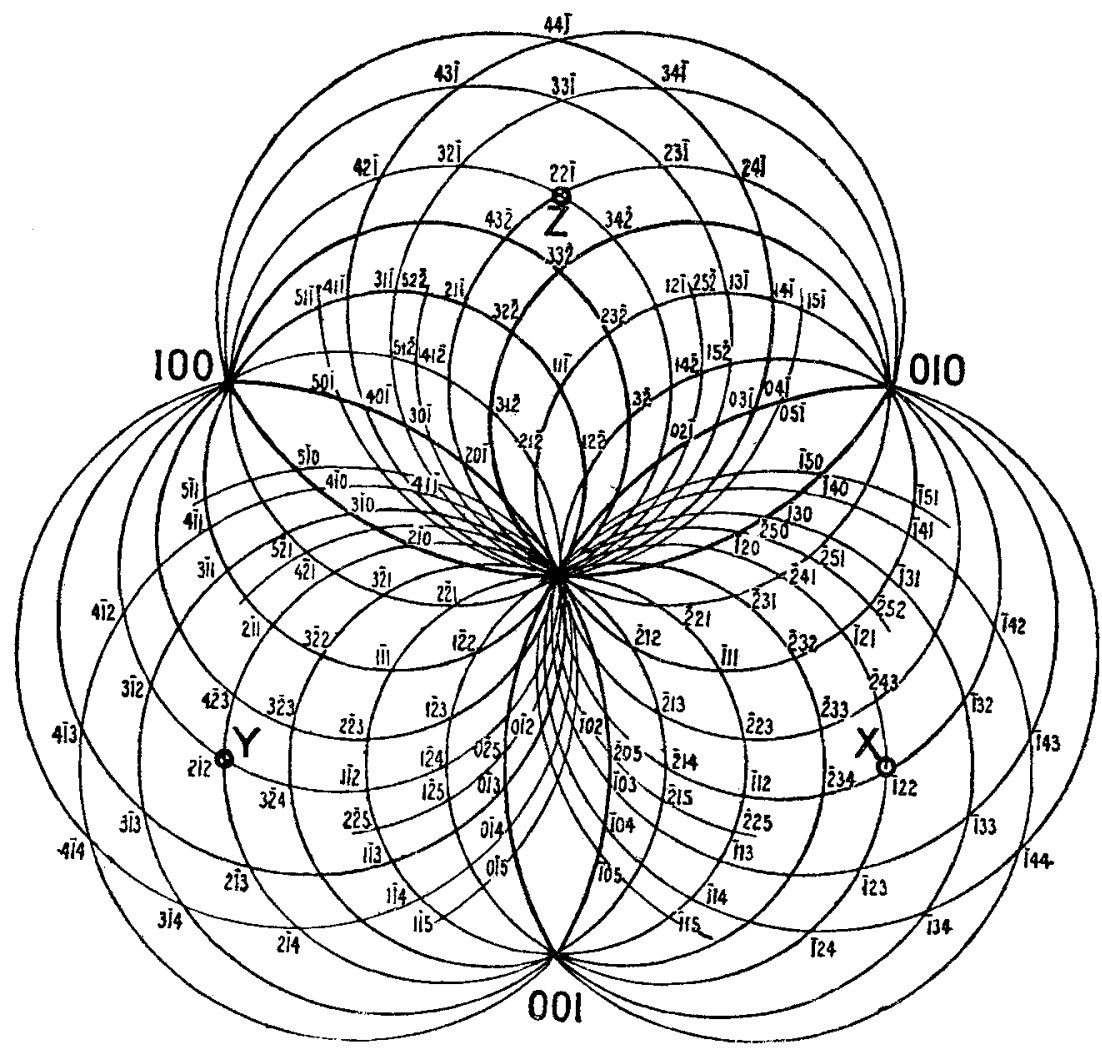

Fig. 5.

Flecke des Kaliumchloridmusters natürlich auf einem doppelt so großem Diagramm liegen, als das Diagramm ist, auf dem die Flecke von Zinkblende angeordnet sind; die Punkte, wo Achsen mit einem Winkel von $90^{\circ}$ die Platte schneiden, liegen gerade zweimal soweit entfernt voneinander, wie die Punkte der Achse mit einem Winkel von $60^{\circ}$.

Die folgende Tabelle 6 wird zeigen, wie verkehrt es ist, die beiden Muster auf das falsche Diagramm zu beziehen. 
Die Struktur einiger Kristalle usw.

Wenn man die Flecke des Zinkblendemusters auf kubische Achsen bezieht, so werden die Indizes viel komplizierter, und das

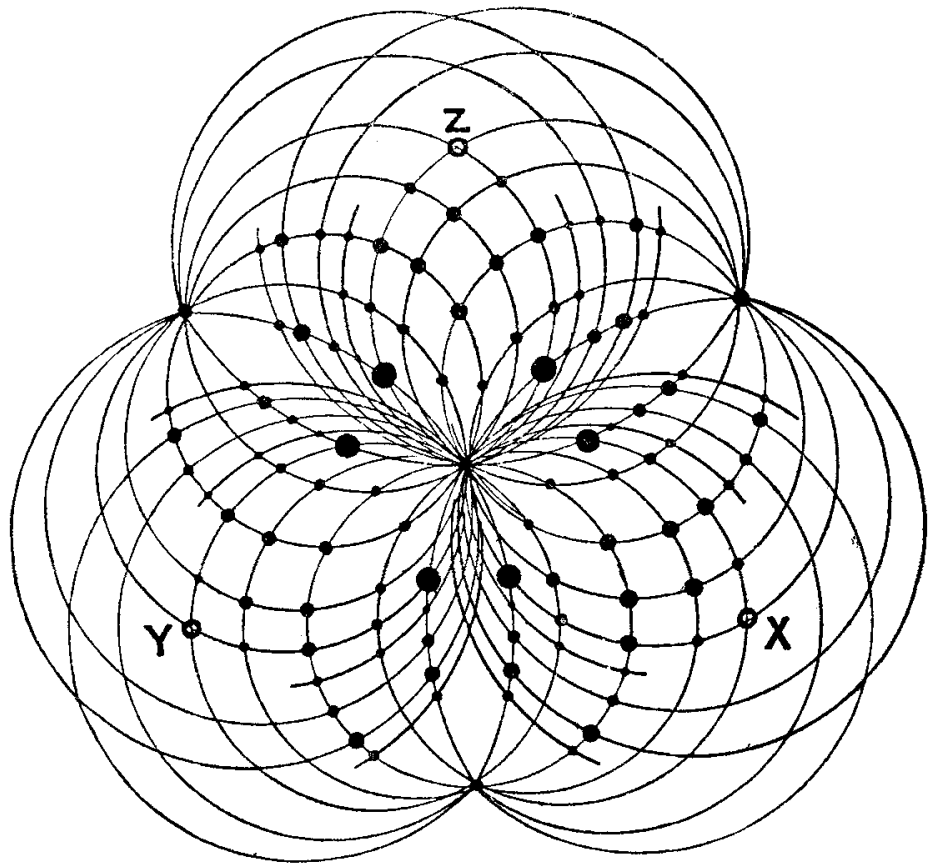

Fig. 6. Zinkblende.

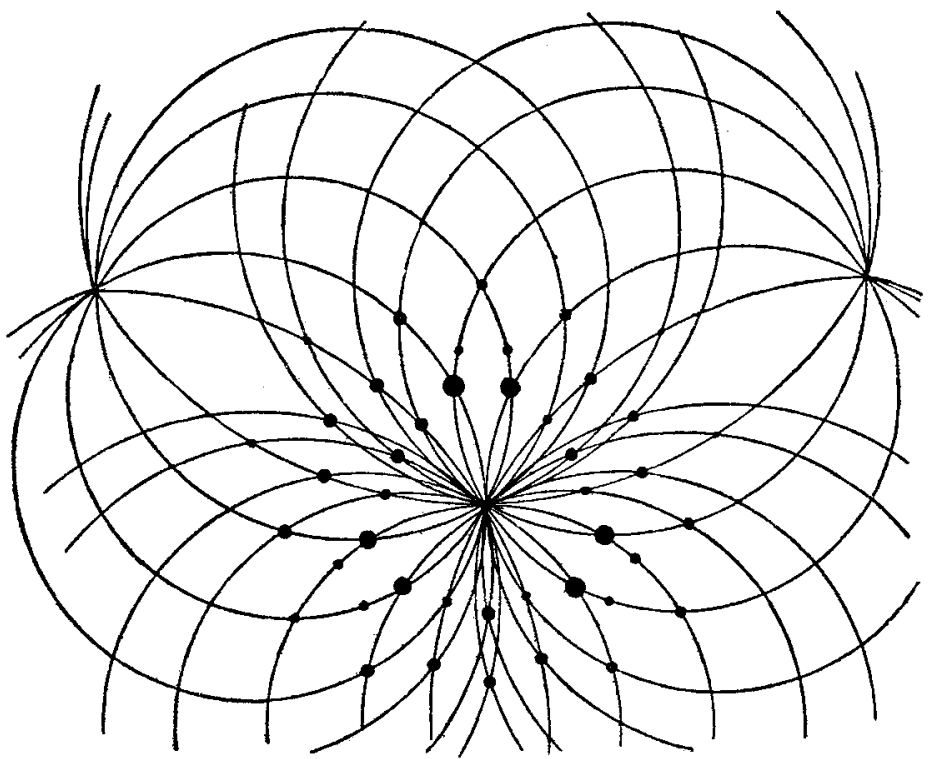

Fig. 7. Kaliumchlorid. 
Muster ist nicht vollständig. Das umgekehrte gilt für das Kaliumchloridmuster, dessen Indizes in dem Falle einfacher werden, wenn man es auf kubische Achsen bezieht. In Tabelle 6 ist eine Liste der Indizes für die Flecke beider Muster aufgestellt und zwar einmal bezogen auf die kubischen Kanten $(A)$, das andere Mal auf die Diagonalen der Würfelflächen $(B)$, d. h. auf drei Kanten des regelmäBigen Tetraeders als Achsen.

Tabelle 6 .

\begin{tabular}{|c|c|c|c|c|c|}
\hline \multicolumn{4}{|c|}{ Zinkblende } & \multicolumn{2}{|c|}{ Kaliumchlorid ${ }^{1}$} \\
\hline$A$ & $B$ & $A$ & $B$ & $A$ & $B$ \\
\hline $\begin{array}{l}\overline{1} 11 \\
332 \\
\overline{5} 53 \\
251 \\
\overline{3} 31 \\
\overline{1} 110\end{array}$ & $\begin{array}{l}010 \\
0 \overline{5} 1 \\
0 \overline{4} 1 \\
0 \overline{3} 1 \\
0 \overline{2} 1 \\
0 \overline{1} 1\end{array}$ & $\begin{array}{l}\overline{1} 11 \\
\overline{3} 75 \\
132 \\
153 \\
\overline{1} 55 \\
021 \\
1 \overline{7} 13 \\
1 \overline{3} 1 \\
3 \overline{5} 1\end{array}$ & $\begin{array}{l}100 \\
51 \frac{1}{1} \\
41 \frac{1}{1} \\
31 \frac{1}{52} \\
52 \frac{1}{1} \\
32 \frac{2}{11} \\
12 \frac{1}{2}\end{array}$ & $\begin{array}{l}31 \overline{1} \\
20 \overline{1} \\
31 \overline{2} \\
11 \overline{1} \\
30 \overline{2} \\
21 \frac{2}{2} \\
41 \overline{1} \\
32 \overline{3}\end{array}$ & $\begin{array}{l}11 \overline{1} \\
21 \overline{1} \\
41 \overline{1} \\
100 \\
31 \overline{2} \\
30 \overline{1} \\
332 \\
50 \overline{2}\end{array}$ \\
\hline
\end{tabular}

Man erkennt, da $B$ bei der Zinkblende die Reihe unter $B$, und beim Kaliumchlorid die Reihe unter $\mathcal{A}$ die einfachen Werte liefert, und eine vollständige Reihe von Indizes über ein gewisses Gebiet vermittelt. Ich denke, es kann kein $Z$ weifel darüber sein, welches Raumgitter in diesen beiden Fällen das richtige ist.

Beim Steinsalz ist es nicht möglich, das Muster als vollkommen charakteristisch für das eine oder das andere Punktsystem zu betrachten. Die Photographien, welche man mit einem dünnen Kristallschnitt von etwa $1 \mathrm{~mm}$ Dicke, parallel zu einer Würfelfäche geschnitten, erhält, zeigt ein Muster, das dem des Kaliumchlorids sehr ähnlich, wenngleich auch in keinem Fall so einfach ist, wie das des letzteren. Es zeigt sich nämlich ein erheblicher Unterschied zwischen den „ungeraden" und den ,geraden“ Ebenen. Eine mit einem $6 \mathrm{~mm}$ dicken Schnitt aufgenommenen Photographie ist denen von Kaliumbromid oder Kaliumjodid ähnlicher, indem die Unter-

I Die Indizes der $A$-Reihe für die Kaliumchloridmuster scheinen auf den ersten Blick nicht einfacher zu sein als die für die $B$-Reihe. Diese ist aber merkwürdig unvollständig. Z. B. kommen $21 \overline{1}$ und $41 \overline{1}$ vor, aber nicht $31 \overline{1}$; ebenso $50 \overline{1}$ und $30 \overline{1}$, aber nicht $40 \overline{1}$; desgleichen $33 \overline{2}$ und $31 \overline{2}$, jedoch nicht 32̄. In der Tat besteht die Tendenz, daB die Summe der Indizes gerade ist, und eine derartige Auswahl der einfuchen Ebenen deutet immer darauf, daB die falschen Achsen gewählt sind. In der $B$-Reihe für $\mathrm{ZnS}$ liegen solche Indizes wie $(52 \overline{2}) \mathrm{zwischen} 31 \overline{1}$ und $21 \overline{1}$. 
schiede zwischen ungeraden und geraden Ebenen mehr ausgesprochen sind. Auf Fig. 8 der Tafel II ist die Photographie eines Steinsalzschnittes von $25 \mathrm{~mm}$ Dicke parallel zur Fläche 100, und in Fig. 9 deren stereographisches Diagramm dargestellt. In den Tabellen auf der folgenden Seite sind die Intensitäten der Flecke bei verschiedener Dicke der Kristalle verzeichnet.

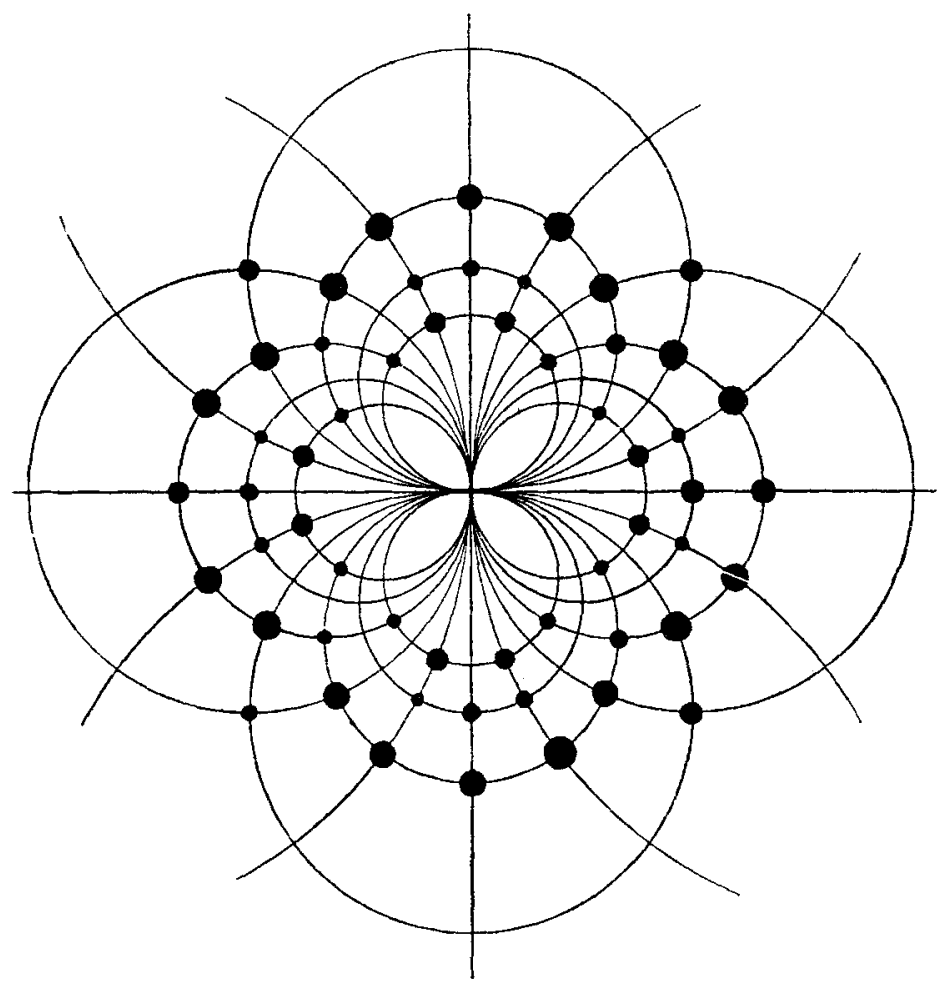

Fig. 9. Steinsalz, $2.5 \mathrm{~mm}$ dick.

Ich denke diese Tabellen, insbesondere die erste, weisen darauf hin, dab das beugende System im Steinsalz gewissermaßen

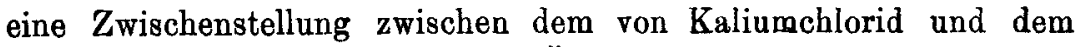
von Kaliumbromid einnimmt. Die Änderung der Intensität der Flecke mit der Dicke des Kristalles ist von Interesse. Es kann als allgemeine Regel angegeben werden, dab Vergrößerung der Kristalldicke die Intensität der inneren Flecke im Vergleich zu der der anderen vermehrt. Dies ist $\mathrm{zu}$ erwarten, denn die inneren Flecke stellen die kürzeren und durchdringenderen Strahlungen dar.

Wenn beim Durchgang durch die Schicht $d x$ des Kristalles ein

Z. anorg. Chem. Bd. 90 . 
Tabelle 7.

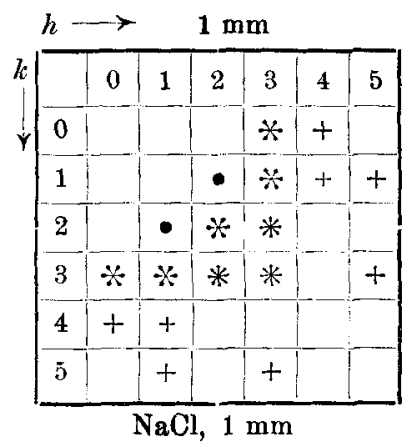

Tabelle 8.

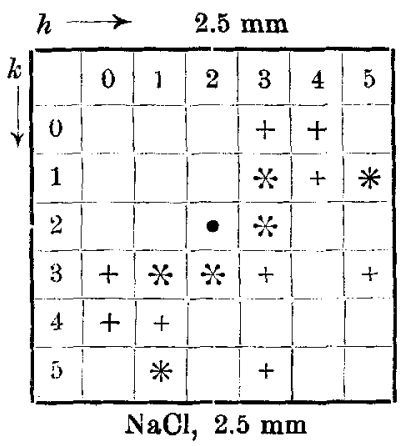

Tabelle 9.

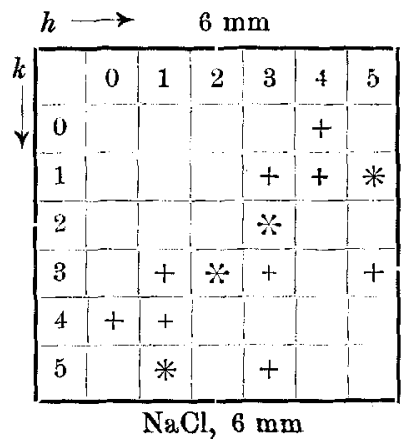

Bruchteil $a d x$ der Strahlung einer bestimmten Wellenlänge reflektiert wird, und wenn der Absorptionskoeffizient der Strahlen im Kristall $k$ ist, dann wird die in einer Schicht des Kristalles von der Dicke $t$ reflektierte Strablung in roher Annäherung sein $\alpha t e^{-k t}$. Dieser Wert erreicht ein Maximum, wenn $t=1 / k$, und da $\alpha$ sehr klein ist, so bedeutet dies, daB zur Erzeugung eines starken Fleckes ein solcher Kristallschnitt benutzt werden muf, der den Bruchteil 1/e der einfallenden Strahlung von dieser Wellenlänge absorbiert; hierdurch würde qualitativ die tatsächlich beobachtete Intensitätsverteilung zwischen den Flecken mit der Änderung der Dicke des Kristallschnittes seine Erklärung finden.

Vergleicht man die wirkliche Natur des beugenden Systems bei diesen Kristallen von Natriumchlorid und von Kaliumchlorid, -bromid und -jodid, so scheint es, daB man zu einer sehr einfachen Erklärung dieses eigenartigen Unterschiedes kommen kann, wenn man berücksichtigt, daB in allen Fällen die Beugung verursacht wird durch zwei verschiedene Atome, und dab die relative Wirk- 
samkeit dieser beiden sich von Kristall zu Kristall ändert. Eine Erklärung dieses Unterschiedes, die nicht eine ähnliche Struktur für die ganze Gruppe der Alkalihalogenide annimmt, würde auBerordentlich unwahrscheinlich sein, denn diese Kristalle sind einander in ibren Eigenschaften sehr ähnlich. Es hat sich aber gezeigt, daB das Raumgitter der beugenden Punkte beim Kaliumchlorid einfach kubisch ist, während es beim Kaliumbromid und Kaliumjodid ein kubisches Gitter mit Punkten auf den Würfelflächen darstellt. Beim Natriumchlorid nimmt das beugende Punktsystem in gewisser Weise eine Mittelstellung zwischen diesen beiden Raumgittern ein.

Wir wollen nun betrachten, von welchen Atomeigenschaften die relative Wirksamkeit abhängen kann. Es ist sicher festgestellt worden, daB die Absorption von X-Strahlen von den relativen Mengen der verschiedenen Elemente abhängt, die im absorbierenden Körper vorhanden sind. Sie ist eine rein additive Eigenschaft des Gewichtes eines jeden Elementes im Kubikzentimeter des Absorptionsmittels, und hängt nicht ron der Art ab, wie die Elemente vereinigt sind. Auch die Absorption homogener X-Strahlen vermehrt sich stetig mit dem Atomgewicht des Absorbens; ausgenommen muB hier werden eine plötzliche Unstetigkeit, die sich in einem starken Abfall des Absorptionskoeffizienten zeigt, wenn das Atomgewicht des absorbierenden Stoffes den Wert des Elementes annimmt, für welches die homogenen X-Strahlen die charakteristische Strahlung bilden. Es finden sich aber keine Unstetigkeiten bei den Absorptionskoeffizienten, die Änderungen von chemischen Eigenschaften der Elemente in der periodischen Tabelle entsprechen, wenn man zu höheren Atomgewichten übergeht. Demnach ist es vorläufig zulässig anzunehmen, daB das Gewicht des Atomes in der Hauptsache seine Wirksamkeit als Beugungszentrum bedingt, und da $B$ zwei Atome von gleichem Gewicht auch gleich wirksam sind. Beim Kaliumchlorid nähern sich die Atomgewichte 39 und 35.5 so sehr, daB diese Elemente als identische Beugungszentren wirken können. Für Steinsalz gilt dies nicht mehr; die Atomgewichte von Natrium und Chlor (35.5 und 23), unterscheiden sich beträchtlich, und so gelangen in das einfache, für Kaliumchlorid charakteristische Muster Komplikationen hinein. Beim Kaliumbromid und beim Kaliumjodid überwiegt ein Atom so stark über die anderen im Atomgewicht, daB das beugende System praktisch nur aus einer Art von Atomen besteht, und das Muster kann wieder auf ein einfaches Raumgitter bezogen werden, das aber eine andere Natur hat als beim Kalium- 
chlorid. Trotzdem haben die Atome der Alkalimetalle und der Halogene in allen diesen Fällen dieselbe Anordnung.

Wir wollen zwischen zwei Arten von beugenden Punkten unterscheiden und sie als schwarz und weiB bezeichnen. Dann müssen die Punkte derartig angeordnet sein, daB

1. die gleiche Anzahl ron schwarz und weiB vorhanden ist,

2. die Anordnung der schwarzen und weißen Punkte, wenn man sie alle zusammen nimmt, die des ersten kubischen Raumgitters ist,

3. die Anordnung der schwarzen allein und der weißen allein die des dritten kubischen Raumgitters darstellt. Eine Anordnung, die dies Ergebnis liefert ist in Fig. 10 wiedergegeben.

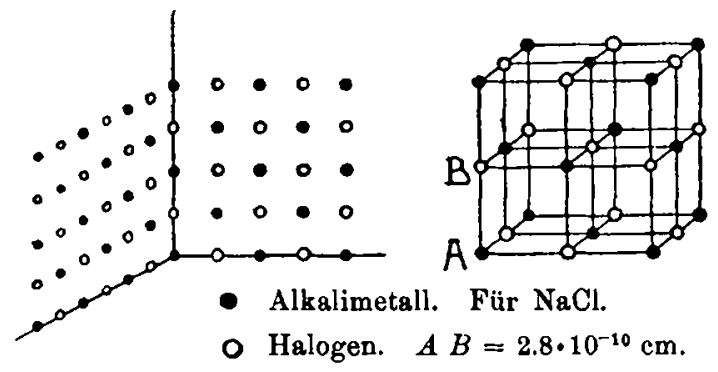

Fig. 10.

In dieser Zeichnung denken wir uns die schwarzen Punkte als Alkalimetall und die weiBen als Halogen oder umgekehrt. Das Raumgitter der weiben ist dasselbe wie das der schwarzen, und zwar jedesmal: Würfel mit Flächenmittelpunkten. Wenn schwarze und weibe Punkte identisch werden, wie beim Kaliumchlorid, so wird das beugende Gitter zum einfachen kubischen Raumgitter.

Der Beweis für diese Anordnung bei den Alkalihalogeniden scheint sehr streng zu sein, aber hierdurch ist durchaus nicht ihre Struktur vollständig aufgeklärt. Denn es bleibt noch die Frage offen, ob ein Atom allein sich an jedem Punkt des Systemes befindet, so daB z. B. beim Steinsalz die schwarzen und die weiben Punkte tatsächlich Natrium und Chlor darstellen, oder ob die Kristallstruktur von komplizierterer Natur ist. Die Analysen der Kristallmuster, die jetzt beschrieben werden sollen, sind in der Absicht ausgeführt worden, die Komplexität der Beugungseinheit von diesen und anderen Kristallen aufzudecken; ihre Ergebnisse scheinen darauf hinzudeuten, daB jedem Beugungsmittelpunkt nur ein A tom entspricht. 
Oben ist gezeigt worden, daB das stereographische Diagramm, welches die Lage der an den Ebenen eines trigonalrhomboedrischen Gitters reflektierten Flecke wiedergibt, - abgesehen rom Mabstab für alle rhomboedrischen Winkel dasselbe ist. Die Vollständigkeit, mit der die Flecke des Zinkblendemusters, dessen Projektion in Fig. 6 dargestellt ist, die Schnittpunkte der Kreise des Diagrammes für das trigonale Gitter wiedergeben, würde den Gedanken nahe legen, da $B$ in allen Fällen, wo Beugungszentren in einem trigonalen Gitter angeordnet sind, das erhaltene Muster im großen und ganzen

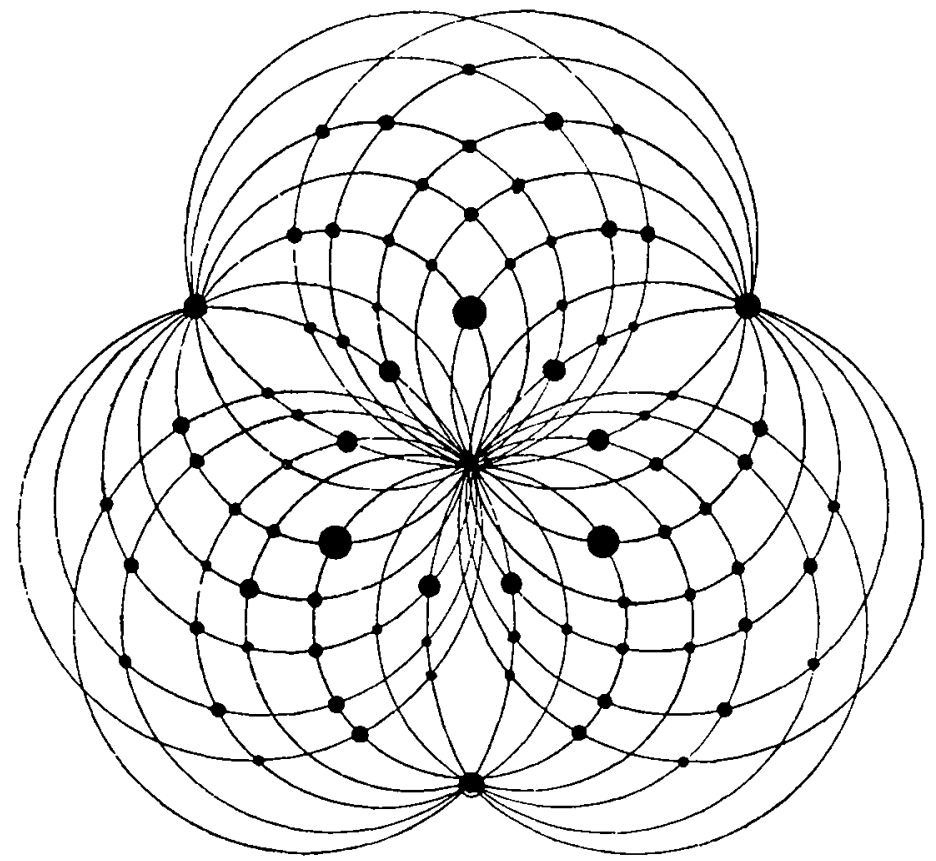

Fig. 12. FluBspat.

dasselbe sein würde. Unter allen Umständen würde dies so sein, wenn der Rhomboederwinkel nicht sehr ron $60^{\circ}$ abweicht. Dab dies wirklich zutrifft, zeigt sich deutlich durch die stereographischen Projektionen in den Figg. 12 und 13. Es sind die Projektionen ron Photographien, die mit Schnitten von FluBspat und Calcit, senkrecht zu einer trigonalen Achse, aufgenommen sind. Die Photographie des Flußspates ist in Fig. 11 auf der Tafel II wiedergegeben. Es ist klar, daB auch in diesem Fall, wie bei der Zinkblende, jede einfache Ebene des Gitters einen Fleck reflektiert, wenn der Ein- 
fallswinkel in einem gewissen Gebiet liegt. Korrespondierende Flecke in den drei Diagrammen unterscheiden sich sehr in der Intensität, aber dies ist auch zu erwarten. Der Punkt, welcher klar gestellt worden soll, ist die Sicherheit, womit die richtigen Achsen des Gitters aus einer Untersuchung des Musters ermittelt werden können. Beim FluBspat wie bei der Zinkblende ist der rhomboedrische Winkel dieser Achsen $60^{\circ}$.

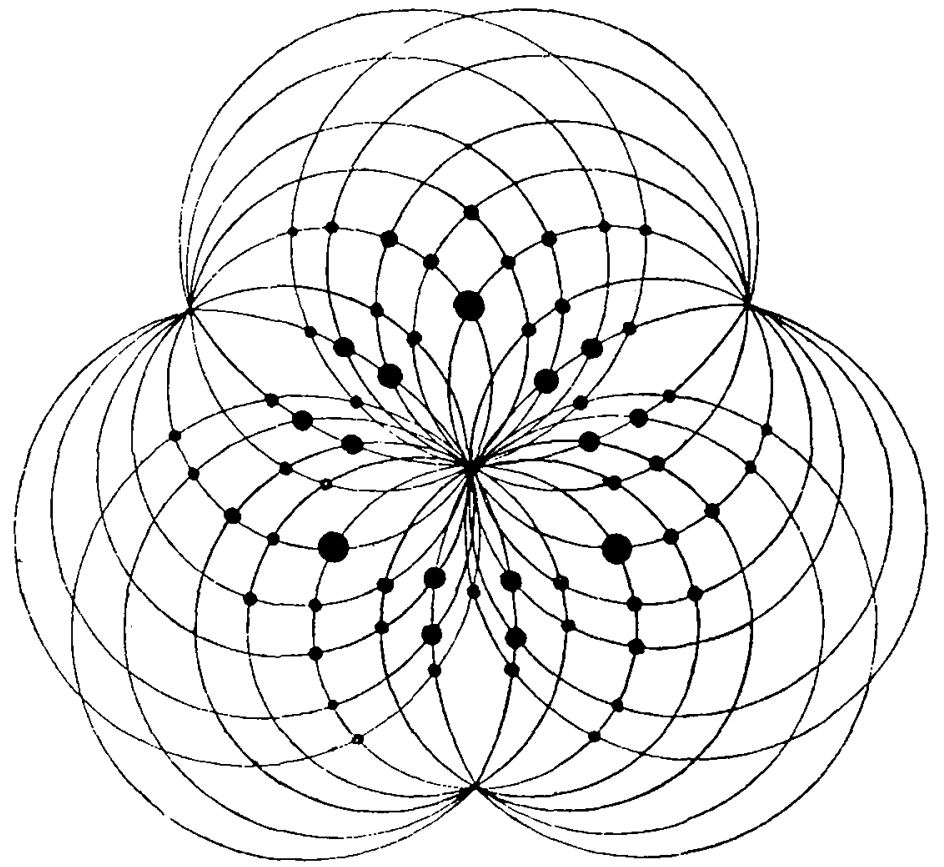

Fig. 13. Calcit.

Beim Calcit ist er etwas gröBer, und eine Berechnung dieses Winkels, sowie ein Vergleich der Orientierung von Kristall und Muster machen es klar, daß die Achsen des Gitters drei Diagonalen von Rhomboederflächen sind, die sich in der stumpfen Ecke des Calcitrhomboeders treffen, nicht aber die Kanten des Rhomboeders, die gewöhnlich als Achsen für die Flächen des Calcitkristalles benutzt werden.

In diesen drei Fällen, bei Zinkblende $(\mathrm{ZnS})$, Flubspat $\left(\mathrm{CaF}_{3}\right)$ und Calcit $\left(\mathrm{CaCO}_{3}\right)$ sind die Beugungsmittelpunkte so in einem Raumgitter angeordnet. Da aber ein Raumgitter eine Anordnung ist, bei dem jeder Punkt zu seinen Nachbarn in genau denselben 
Beziehungen steht, wie jeder andere Punkt, so würde es unmöglich sein, komplizierte Molekel in Raumgittern anzuordnen, wenn nicht nur ein Punkt in jeder Molekel wirksam ist. Es ist schwierig, den SchluB zu vermeiden, daB die Molekel als einzelner Punkt wirkt, weil sie in jedem Falle ein Atom von viel gröBerem Atomgewicht enthält als die anderen. Ein Vergleich mit einem Falle, wo dies nicht so ist, bietet sich bei dem Steinsalzmuster von dreizähliger Symmetrie in Figg. 14 und 15. Man erkennt, wie wenig es zum

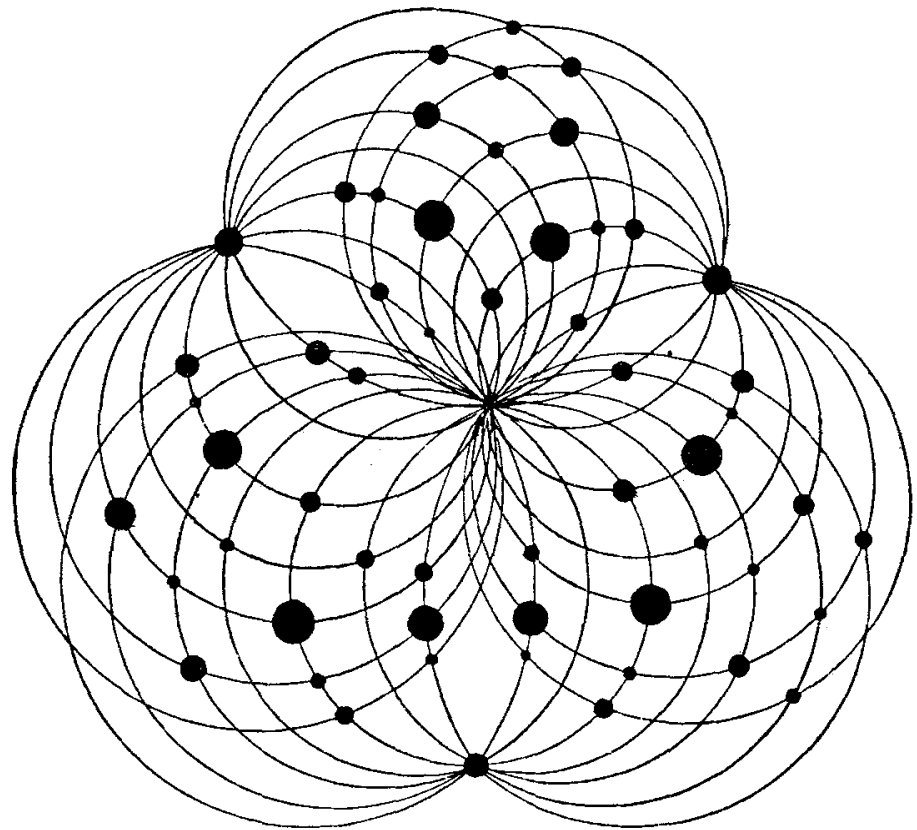

Fig. 14. Steinsalz.

Diagramm paBt. Es ist ein gewisser Mangel an Symmetrie in den Figuren, der nicht verwechselt werden darf mit dem Mangel an Übereinstimmung. Sein Muster nimmt in der Tat eine Mittelstellung ein zwischen dem, das charakteristisch ist für Achsen mit rhomboedrischen Winkel von $60^{\circ}$ und dem für Achsen mit $90^{\circ}$, genau wie es der Fall war mit seinem Muster von vierzähliger Symmetrie.

Nachdem einmal die Natur des einfachen Beugungsgitters in diesen drei Fällen sowie bei den Alkalihalogeniden festgestellt war, wird der letzte Schritt der Beweisfuhrung dafür, daB ein einzelnes schweres Atom einem jeden Beugungszentrum zuzuerteilen ist, weiter 
unten geführt werden durch einen Vergleich des Maßstabes ihrer Gitter, d. h. durch einen Vergleich der Seitenlänge des Elementarparallelepipedes für verschiedene Kristalle. Da die Beugung durch die schweren Atome verursacht wird, und da nur eins von ihnen in jeder Molekel vorhanden ist, so bedeutet dieser SchluB, wenn er zutrifft, daB zu jedem Parallelepided des Gitters eine Molekel gehört.

In der der Royal Society im April 1913 vorgetragenen Mitteilung ${ }^{1}$ wurde eine Methode beschrieben zur Analyse der Strahlung aus X-Strahlröhren durch Reflexion derselben von der Fläche eines Kristalles und Messung der durch das reflektierte Bündel hervorgerufenen Ionisation.

Der für diesen Zweck benutzte Apparat war einem Spektrometer ähnlich; der Kristall stand auf einem drehbaren Tisch im Mittelpunkt des Instrumentes und wurde bestrahlt von einem schmalen Bündel X-Strablen, die von einem Kollimatorspalt ausgingen. Das reflektierte Bündel wurde in einer, wie ein Spektrometerfernrohr aufgestellte Ionisationskammer aufgefangen und gemessen. Mit diesem Instrument war es möglich, die Stärke des reflektierten Bündels zu messen, wenn ein angenähert konstantes Bündel von Strahlen auf die Fläche des Kristalles bei verschiedenen Einfallswinkeln auftraf. Das Ergebnis der Messungen mit einer Kristallfäche kann in einer Kurve wiedergegeben werden, in welcher die Stärke des reflektierten Bündels gegen den Spiegelungswinkel, unter dem die Strahlen auf den Kristall fallen, aufgetragen ist. Bei den meisten Kristallen zeigt eine derartige Kurve bei allen Winkeln eine allgemeine Reflexion der Strahlen und die Reflexion ist viel stärker bei den mehr spiegelnden Winkeln. Über diese allgemeine Reflexion lagert sich in bestimmten, sehr scharf definierten Winkeln eine besondere Reflexion von sehr großer Intensität im Vergleich mit der allgemeinen Reflexion. Diese besondere Reflexion zeigt sich in Form sehr ausgeprägter Spitzen auf der Kurve, und die GröBe der Ionisation in der Kammer wird etwa $20 \mathrm{mal}$ so groB, wenn die Kammer durch das Gebiet einer Spitze geführt wird, und fällt dann wieder auf ihren normalen Wert. Die ganze Erscheinung spielt sich zwischen zwei Stellungen des Apparates ab, die sich nur um einen Grad unterscheiden.

Die Ergebnisse, die wir mit verschiedenen Kristallen erbielten, deuteten auf die Existenz ron wenigstens drei Komponenten des

I W. H. Brage und W. L. Braga, Proc. Roy. Soc, Juli 1913. 
$\mathrm{X}$-Strahlenbündels von verschiedener Wellenlänge, die von einem Kristall reflektiert werden, wenn die Bedingung

$$
n \lambda=2 d \sin \Theta
$$

von der Komponente und dem fraglichen Kristall erfüllt werden. Hier ist $\lambda$ die Wellenlänge, $\Theta$ der Spiegelungswinkel und $d$ die Entfernung zwischen aufeinanderfolgenden Ebenen der Kristallstruktur, parallel zur reflektierenden Ebene. Die Zahl $n$ gibt die „Ordnung““ der Reflexion an, denn die verschiedenen Spitzen, die als zur selben homogenen Strahlung zugehörig erkannt werden können durch die Identität ihrer Absorptionskoeffizienten, treten bei einer Reihe ron Winkeln auf, deren Sinus im Verhältnis $1: 2: 3$ stehen.

Man kann hier möglicherweise einwenden, daB bei der vorhergehenden Besprechung der Interferenzmuster das Spektrum der einfallenden Strahlung kontinuierlich angenommen ist, und daB die Einfachheit der Photographie von Kaliumchlorid diese Ansicht stützt; hier dagegen wird der direkte Beweis für die Existenz homogener Komponenten in der Strahlung erbracht. Die Schwierigkeit verschwindet aber, wenn man die Wellenlängen, die den Flächen des Interferenzmusters und den Spitzen der Kurve zukommen, vergleicht. Z. B. wird beim Kaliumchloridmuster von vierzähliger Symmetrie (Fig. 2) der durch die längsten Wellen gebildete Fleck an den Ebenen (2 21 1) reflektiert; und wenn $a$ die Seite des elementaren Würfels des Gitters dieses Kristalles ist, so wird die Wellenlänge, die man dem Fleck zuschreiben muB, nach der Formel

$$
\lambda=2 d \sin \Theta=2 a \sin \Theta=a \times 0.22 \text {. }
$$

Andererseits wird die Spitze, die der homogenen Strahlung von kürzester Wellenlänge entspricht, von der Fläche (100) desselben Kristalles in einem Winkel von $8.4^{\circ}$ reflektiert und entspricht ungefähr einer Wellenlänge von $0.29 \mathrm{a}$. Die Flecke des Musters entsprechen so einer Gegend des Spektrums, die ganz an einer Seite der Spitzen der Reflexionskurve liegt, und im allgemeinen ist dies für alle Muster zutreffend. Die Reflexionskurve unterstützt durchaus die Annahme über die kontinuierliche Natur des Spektrums der Strahlung, das die Flecke liefert. Denn bei allen Winkeln, die kleiner sind als $10^{\circ}$, findet eine starke Reflexion statt, die nur allmählich geringer wird, wenn der Spiegelungswinkel sich vergröBert. Schon allein die Tatsache, daB bei den meisten Photographien die einfallenden Strahlen einer Symmetrieachse parallel sind, machen 
es sicher, daB keine wichtigen Ebenen vorkommen, die einen derartigen Winkel mit den primären Strahlen bilden, daB die homogenen Komponenten reflektiert werden könnten.

Es ist möglich, dieselben drei Spitzen, die als $A, B, C$ bezeichnet werden sollen, in den Kurven, die für die Reflexion an den Flächen fast aller untersuchter Kristalle gezeichnet worden sind, wieder zu finden. Sie haben immer ungefähr dieselbe relative Höhe, jede hat einen charakteristischen Koeffizienten, und sie sind auf den Kurven in derselben Weise verteilt. Es scheint kein $Z$ weifel zu sein, daB drei Linien ${ }^{3}$ ) im Spektrum der einfallenden Strahlung vorhanden sind, die diese Spitzen hervorrufen.

Wenn dies so ist, so hat man ein Mittel, mit einiger Genauigkeit das Verhältnis der Werte von $d$ zu finden, also des Abstandes zwischen aufeinanderfolgenden Ebenen der Struktur für verschiedene Kristalle und verschiedene Flächen desselben Kristalles.

Die Winkel, unter denen diese Spitzen von den verschiedenen Flächen eines Kristalles, sowie von den Flächen verschiedener Kristalle reflektiert werden, liefern so einen tiefen Einblick in die Kristallstruktur und in der Tat vermitteln sie gerade die Angabe über die Struktur, die das Interferenzmuster nicht gibt. Denn mit ihrer Hilfe können die Dimensionen der Gitter verschiedener Kristalle genau verglichen werden. Das Interferenzmuster liefert nur AufschluB über die Natur der Gitter.

Eine Analyse der Ergebnisse, die man erhielt, als verschiedene Flächen desselben Kristalles zur Reflexion der X-Strahlen im Spektrometer benutzt wurden, wird zeigen, wie dieser Vergleich aus. geführt wird.

In den Reflexionskurven von den drei Hauptflächen des Steinsalzkristalles (100), (110), (111), von denen zwei in Fig. 16 dargestellt sind, kommen die Spitzen für jede Fläche vor, aber bei verschiedenen Winkeln. Aus der Gleichung

$$
\lambda=2 d \sin \Theta,
$$

wo $\Theta$ der Winkel für die Spitze der ersten Ordnung in jedem Falle ist, haben wir für diese drei Flächen

$$
d_{(100)}: d_{(110)}: d_{(111)}=\frac{1}{\sin \Theta_{(100)}}: \frac{1}{\sin \Theta_{(110)}}-\frac{1}{\sin \Theta_{(111)}},
$$

1 Durch Anwendung enger Spalte im Reflexionsapparat konnte der Beweis für die Existenz von mehr als drei Linien erbalten werden; die Spitzen $B$ und $C$ sind in Wirklichkeit doppelt. S. Fig. 14. 
wo $d_{(100)}$ der Abstand zwischen Ebenen parallel der Fläche (100), $\Theta_{(100)}$ der Winkel ist, unter dem die stärkste Spitze von derselben Fläche reflektiert wird.

Die Winkel für diese drei Flächen sind $11.4^{\circ}, 16.0^{\circ}, 9.8^{\circ}$. Dies liefert das Ergebnis

während

$$
d_{(100)}: d_{(110)}: d_{(111)}=1: 0.718: 1.16,
$$

$$
1: \frac{1}{\sqrt{2}}: \frac{1}{\sqrt{3}}=1: 0.707: 1.15
$$

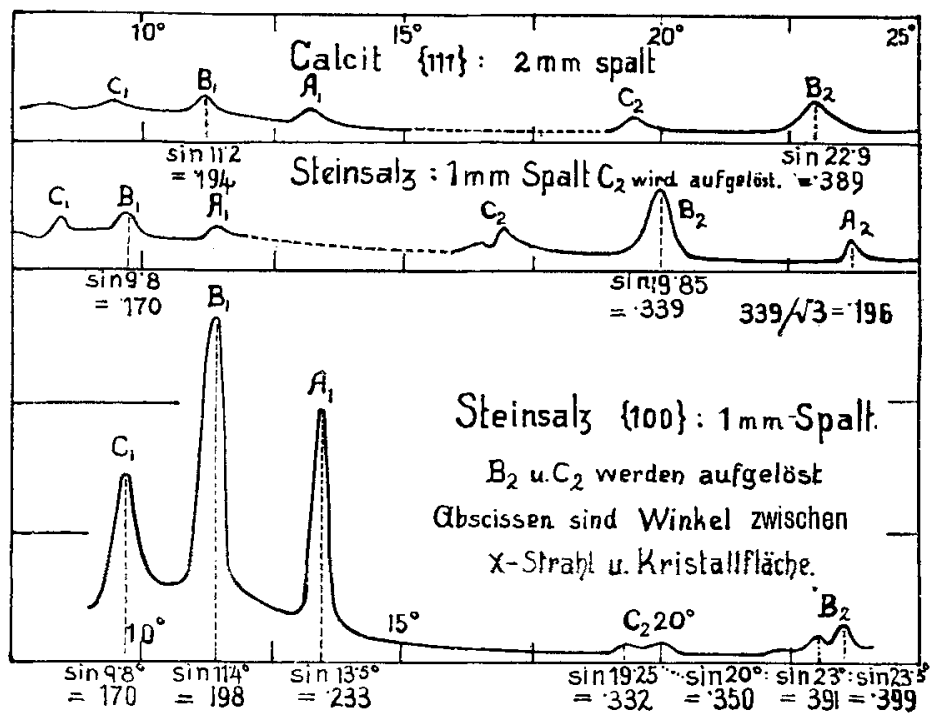

Fig. 16.

Es sind dies die theoretischen Beziehungen für das kubische Raumgitter mit Flächenmittelpunkten. Hierdurch ist jedoch nicht der ganze Finblick, den man aus einer Untersuchung dieser drei Kurven erhalten kann, erschöpft; sie werden vielmehr weiter unten noch sorgfältig analysiert werden. Im Augenblick ist es ausreichend, darauf hinzuweisen, welch starke Gründe für die Annahme vorhanden sind, daß die Abstände $d$ für die verschiedenen Flächen genau verglichen werden können, wenn man die Winkel aufsucht, bei denen diese Spitzen auftreten.

Wir haben gesehen, daB die von Kaliumchlorid, Zinkblende, FluBspat und Calcit gelieferten Muster der Beugung durch Punkte eines Raumgitters zugeschrieben werden können. Es ist nun wünschenswert, die Dimensionen des Gitters dieser Kristalle zu ver- 
gleichen; da die absoluten Werte für die Wellenlänge $\lambda$ der Strahlung, die die Spitzen liefert, bisher unbekannt ist, so sollen die Dimensionen des Gitters auf die Größe $\lambda$ als Einheit bezogen werden.

Da nun sowohl die Form, wie auch die Dimensionen (bezogen auf $\lambda$ ) des elementaren Parallelepipedes für die Raumgitteranordnung der beugenden Mittelpunkte dieser Kristalle bekannt sind, ist es möglich, das Volumen dieses Parallelepipedes zu berechnen; es ist dies dasjenige Volumen, welches mit jedem Punkt des Gitters verbunden ist; es ist der reziproke Wert der Anzahl von Punkten im Einheitsvolumen. Multiplikation dieses Volumens mit der Dichte des Kristalles liefert die Masse, die mit jedem Beugungsmittelpunkt verbunden ist, und es ist zu erwarten, daß der Vergleich dieser Massen für verschiedene Kristalle einen Anhaltspunkt dafür liefern wird, ob der Mittelpunkt aus Atomen, Molekeln oder Gruppen von Molekeln besteht. Die Ergebnisse dieser Berechnungen für die verschiedenen Kristalle sind in der folgenden Tabelle zusammengestellt.

In dieser bedeutet:

$\Theta=$ Spiegelungswinkel der $B$-Spitze erster Ordnung;

$d=$ Abstand zwischen den Ebenen, die der untersuchten Fläche parallel sind;

$V=$ Volumen des Elementarparallelepipides berechnet, aus diesem Wert von $d$ und einer Kenntnis von der Natur des Gitters; $\varrho=$ Dichte des Kristalles;

$M=$ Molargewicht des Stoffes.

Tabelle 10.

\begin{tabular}{|c|c|c|c|c|c|c|c|c|}
\hline Kristall & Gitter & Fläche & in $^{0}$ & $\frac{d}{\lambda}$ & $\frac{V}{\lambda^{3}}$ & $\varrho$ & $M$ & $\frac{V g}{\lambda^{3} M}$ \\
\hline $\mathrm{KCl}$ & afuch kub & $\begin{array}{l}(100) \\
(111)\end{array}$ & $\begin{array}{l}10.2 \\
18.0\end{array}$ & \begin{tabular}{|l|}
2.86 \\
1.62
\end{tabular} & $\begin{array}{l}23.4 \\
22.2\end{array}$ & 1.97 & 74.5 & 0.605 \\
\hline Steinsalz, $\mathrm{NaCl}$ & $\begin{array}{c}\text { Kubisch, mit Flächen- } \\
\text { mittelpunkten }\end{array}$ & $\begin{array}{l}(100) \\
(110) \\
(111)\end{array}$ & $\begin{array}{r}11.4 \\
16.0 \\
9.8\end{array}$ & $\begin{array}{l}2.53 \\
1.82 \\
2.95\end{array}$ & $\begin{array}{l}32.5 \\
33.9 \\
33.5\end{array}$ & 2.15 & 58.5 & 1.22 \\
\hline Zinkblende, ZnS & & $(110)$ & 16.5 & 1.76 & 30.8 & 4.06 & 97.0 & 1.28 \\
\hline FluBspat, $\mathrm{CaF}_{2}$ & d & $\begin{array}{l}(100) \\
(111)\end{array}$ & $\begin{array}{l}11.7 \\
10.3\end{array}$ & $\begin{array}{l}2.46 \\
2.79\end{array}$ & $\begin{array}{l}29.8 \\
28.3\end{array}$ & 3.18 & 78.0 & 1.18 \\
\hline Calcit, $\mathrm{CaCO}_{3}$ & Rhomboedrisch & $\begin{array}{l}(100) \\
(111)\end{array}$ & $\begin{array}{l}10.5 \\
11.2\end{array}$ & $\begin{array}{l}2.74 \\
2.60\end{array}$ & 44.8 & 2.71 & 100.0 & 1.22 \\
\hline Pyrit, $\mathrm{FeS}_{2}$ & $\begin{array}{c}\text { Kubisch, wit Flächen- } \\
\text { mittelpunkten (?) }\end{array}$ & $(100)$ & 12.1 & 2.39 & 27.3 & 5.03 & 120.0 & 1.15 \\
\hline
\end{tabular}

Die letzte Spalte enthält den Wert von $V \varrho / \lambda^{3} M$, die Masse, die mit jedem Beugungszentrum vereinigt ist, geteilt durch das-

' Das Interferenzmuster dieses Kristalles ist bisher noch nicht erhalten worden. 
Molargewicht der Substanz. Diese Menge ist demnach proportional der Anzahl der mit jedem Mittelpunkt vereinigten Molekel. Für alle diese Kristalle, ausgenommen Kaliumchlorid, ist diese Größe innerbalb der Versuchsfehler ${ }^{1}$ die gleiche, woraus hervorgeht, daB bei all diesen Kristallen die Anzahl der Molekel, die mit jedem Beugungsmittelpunkt vereinigt sind, dieselbe ist. Berücksichtigt man die sehr verschiedene Konstitution dieser Kristalle, so scheint dies darauf hinzudeuten, daB nur 1 Molekel auf jeden Beugungsmittelpunkt trifft; und da bei Zinkblende, FluBspat und Calcit die Molekel nur e in schweres Atom enthält, so kommt man zu dem SchluB, daB das Raumgitter, welches durch die Beugungsmuster angezeigt wird, dem Gitter entspricht, welches die einzelnen Zink- oder Calciumatome dieser Kristalle bilden.

Kaliumchlorid macht von dieser Regel augenscheinlich eine Ausnahme, denn es hat einen Wert für $V \varrho / \lambda^{3} M$, der halb so groB ist, wie bei den anderen Kristallen. Die Ursache hierfür ist klar, wenn man sich daran erinnert, daB in Kaliumchlorid zwei Atome von nahezu gleichem Atomgewicht vorhanden sind. Jede Molekel bildet dann zwei Beugungszentren, die in dem einfachen kubischen Raumgitter angeordnet sind. Die mit jedem Beugungsmittelpunkt vereinigte Masse ist nicht die einer Molekel, sondern nur die Hälfte daron und wieder bilden die einzelnen Atome, diesmal aber zwei Arten, die Punkte des beugenden Raumgitters.

Es ist klar, daB die hier angeführten Gründe keinen Anspruch haben, ein vollständiger Beweis für diesen Punkt zu sein. Es wäre z. B. begreiflich, daB bei all diesen Kristallen zufälligerweise gerade die Molekel zu Gruppen von je vier zusammentreten, und daB diese Gruppen die Beugungszentren bilden. Es ist leicht, eine solche Anordnung für die Alkalihalogenide sich vorzustellen, und in der Tat ist dies die Anordnung, die nach der Theorie der engsten Packung von POPE und BanLow allen solchen binären Verbindungen zuerteilt werden muB. Diese Anordnung würde in zufriedenstellender Weise die Muster und die Spitzenbeziehungen bei Steinsalz, Zinkblende, Kaliumbromid und Kaliumjodid wiedergeben, denn die schwarzen und die weißen Mittelpunkte der Zeichnung von Fig. 8 werden dargestellt durch Tetraeder, die aus vier Kugeln bestehen, welche den Atomen beider Arten entsprechen. Dies würde jedoch

1 Es muB daran erinnert werden, daB bei der Berechnung dieser Größe jeder prozentische Fehler im Werte von $\sin \Theta$ verdreifacht wird, weil sin $\Theta$ in die dritte Potenz erhoben wird. 
auch bedingen, daB sich die Calciumatome im Calcit zu vieren gruppieren, und es bietet beträchtliche Schwierigkeiten, eine derartige Anordnung sich auszumalen. Eine ähnliche Schwierigkeit tritt beim FluBspat auf. Kaliumchlorid kann auch kaum für eine solche Anordnung in Anspruch genommen werden, wenn man zugibt, $\mathrm{daB}$ in dieser Substanz Kalium und Chlor fast identisch auf die X-Strahlen wirken, denn die Atome bilden bei der engsten Packung das Gitter des Würfels mit Flächenmittelpunkten, während das Beugungsmuster charakteristisch ist für das einfache Würfelgitter. Es sind viel mehr Vergleiche von Kristallen notwendig, um diesen Punkt zu bestätigen, inzwischen soll angenommen werden, daB die einfache Struktur die Wahrheit richtig darstellt, und daB die Beugungsmittelpunkte einfache Atome sind.

Wir haben gesehen, wie der Vergleich der Reflexionswinkel einer Spitze von verschiedenen Kristallfächen desselben Kristalles über die Raumgitterstruktur des Kristalles Aufschluß liefern kann. Weitere Einblicke erhält man durch Untersuchung der Abmessungen dieser Spitzen. Z. B. sind die Kurven für zwei ron den drei Hauptflächen (100), (110), (111) ron Steinsalz in Fig. $16^{1}$ dargestellt, und ein Blick auf diese Kurven zeigt die sehr erheblichen Unterschiede zwischen den Kurven für die Fläche (100) und denen der Fläche (111). Die Kurve für 100 zeigt sehr ausgesprochene Spitzen erster Ordnung, viel kleinere Spitzen zweiter Ordnung und kaum Andeutungen von Spitzen der dritten Ordnung. Die Kurve für (111) andererseits zeigt die Spitzen zweiter Ordnung viel stärker als die der ersten Ordnung.

Dieser Unterschied der Kurven entspricht einem Unterschied in der Natur der Ebenen, die parallel zu diesen Kristallflächen liegen. Bei der Anordnung der schwarzen und weißen Punkte in Fig. 8 erkennt man, daß die aufeinanderfolgenden Ebenen parallel zu (100) die gleiche Anzahl von schwarzen und weißen Punkten enthalten; das gleiche gilt für die Ebenen (110), die auch eine starke Reflexion von erster Ordnung liefern. Die Ebenen parallel mit (111) andererseits enthalten abwechselnd nur schwarze und nur weiBe Punkte. Die schwarzen Punkte allein bilden ein Gitter mit Flächenmittelpunkten, für welches die (111)-Ebenen weiter voneinander entfernt sind als die (100)-Fbenen im Verbältnis $2 / \sqrt{3}$. So

1 Für die experimentellen Beweise zur Stütze dieses Teiles meiner Beweisführung bin ich meinem Vater zu Dank verpflichtet. 
entsprechen. die kleinen Spitzen erster Ordnung die von der (111)Ebene des Steinsalzes reflektiert werden, einer Periodizität der schwarzen Ebenen allein. die parallel (111) liegen und die schweren Chloratome enthalten. Die Gegenwart der Ebenen mit den Natriumatomen zwischen den anderen Ebenen zerstört nicht vollständig diese Reflexion erster Ordnung, aber sie wirken doch stark nach dieser Richtung, während natürlich die Reflexion der zweiten Ordnung verstärkt wird, so daß eine große Spitze von zweiter Ordnung entsteht. Dies erklärt das anormale Verhältnis der Spitzen verschiedener Ordnung bei Reflexion von den Flächen (111), im Vergleich mit den Reflexionen von den Flächen (100) und (110).

In Übereinstimmung hiermit wurde gefunden, daB bei Ersatz von Natrium durch Kalium die erste Spitze erster Ordnung bei Reflexion von der (111)-Fläche so klein wird, daB sie nicht aufgefunden werden kann; die Kurve von (111) beim Sylvin scheint eine Spitze der ersten Ordnung zu haben bei einem Winkel, welcher Flächen entspricht, die $1 / \sqrt{3}$ soweit voneinander entfernt sind, wie die zu (100) parallelen Ebenen. In der Tat liegen die Spitzen, wo sie für ein einfaches kubisches Raumgitter liegen sollten.

Diese Begründung läßt sich folgendermaßen zusammenfassen. Die Anordnung der schweren Atome dieser Kristalle (Kaliumchlorid mit seinen zwei gleichschweren Atomen ist eine Ausnahme) ist diejenige des Raumgitters, das das Skelett des Kristalles bildet, so daB eine Molekel mit einem schweren Atom sich an jedem Punkt des Gitters befindet. Die Spitzen erster Ordnung auf den Reflexionskurven finden sich in Lagen, die die Theorie für dies Raumgitter liefert. Aber die relativen GröBen der Spitzen erster und zweiter Ordnung auf jeder Kurve werden beeinfluBt durch die Lage der leichteren Atome in der Kristallstruktur. Wenn diese leichteren Atome so angeordnet sind, daB sie in den Ebenen des Raumgitters der schweren Atome parallel zur untersuchten Fläche liegen, dann kann man die Reflexionskurve als normal bezeichnen; sie hat groBe Spitzen der ersten und kleine Spitzen der zweiten Ordnung. Wenn die leichteren Atome in Ebenen angeordnet sind, die auf dem halben Wege zwischen den Ebenen des Gitters liegen, so werden die Spitzen erster Ordnung verkleinert und die der zweiten Ordnung verstärkt.

Die Kurven von den Flächen (100) und (111) beim FluBspat zeigen diese Wirkung in sehr ausgesprochener Weiso (Fig. 17). Dieser Kristall hat als Skelett das kubische Gitter mit Flächenzentren, und die Punkte des Gitters werden eingenommen von den 
Calciumatomen; die Fluoratome sind so angeordnet, daB die Reflexion an den (111)-Ebenen nunmebr normalen Typus zeigt, in starkem Gegensatz zu den Kurven für Steinsalz. Andererseits ist die ReHexion erster Ordnung ron der Fläche (100) fast verschwunden. Die Fluoratome müssen so angeordnet sein, daB sie auf oder in der Nähe der (111)-Ebenen des Fundamentalgitters liegen, nicht auf den Ebenen (100) wie die Natriumatome des Steinsalzes. Die Calcitkurve in Fig. 16 zeigt, daB für diesen Kristall die (100)-Ebenen normale Reflexion liefern, während die Kurve für die Fläche (111)

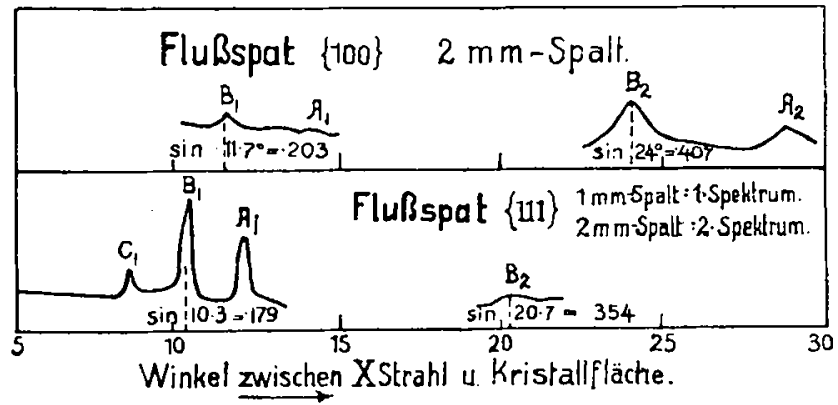

Fig. 17.

sehr ähnlich ist der Kurve für die entsprechende Fläche beim Kochsalz. Es ist $z u$ hoffen, daB die Untersuchung der Reflexion ron verschiedenen Flächen all dieser Kristalle zur Aufdeckung der genauen Lagen der leichteren Atome in der Kristallstruktur führen wird. Bisher sind die experimentellen Ergebnisse noch sehr unvollständig. Die bis jetzt erhaltenen Ergebnisse scheinen mit einiger Sicherheit die Anordnung der schweren Atome dieser einfachen Kristalle festzulegen, und bei den Alkalihalogeniden kann man hoffen, daB die Lagen, die man den Atomen von beider Art beigelegt hat, in jedem Fall enge Annäherung an die Wahrheit bieten. Eine geringe symmetrische Verzerrung der Anordnung, die die Kristallsymmetrie vermindern würde, könnte keinen EinfluB haben auf die hier erzielten Resultate.

Die Analyse von Kristallstrukturen, die hier mitgeteilt wurde, war ursprünglich unternommen worden mit der Absicht, die absoluten Wellenlängen in Zentimetern an der bomogenen, von einer X-Strablröhre ausgehenden Strahlung aufzufinden. Die Lagen der Spitzen auf den Kurven lieferten die Wellenlängen der entsprechenden Strahlungen bezogen auf die Dimensionen des Kristallraumgitters. So lange die Komplexităt der mit jedem Punkt des Gitters 
verbundenen Einheit unbekannt ist, kann die absolute Wellenlänge nicht berechnet werden. Wenn die Anordnung, die hier den Alkaljhalogeniden zuerteilt wurde, richtig ist, können die Dimensionen des Gitters in Zentimetern angegeben werden, denn die mit jedem Mittelpunkt des Gitters in Verbindung stehende Masse läBt sich berechnen aus der bekannten Masse des Wasserstoffatoms in Grammen.

Für Steinsalz ist die Masse von 1 Molekel $\mathrm{NaCl}=\mathbf{5 8 . 5} \times \mathbf{1 . 6 4}$ $\times 10^{-24}$; demnach ist $58.5 \times 1.64 \times 10^{-24}=V \varrho=33.3 \times 2.15 \times \lambda^{3}$

$$
\lambda^{3}=1.34 \times 10^{-24}, \lambda=1.10 \times 10^{-8} \mathrm{~cm} \text {. }
$$

Aus dem Wert von $\lambda$ und dem für $d / \lambda$ aus Tabelle 10 können die Dimensionen des Gitters für jeden Kristall dieser Tabelle berechnet werden.

\section{Zusammenfassung.}

Für eine Anzahl einfacher Kristalle kann das Interferenzmuster zurückgeführt werden auf die Beugung einer „weiBen“ Strahlung durch eine Anzahl von Punkten in einem Raumgitter. Jeder dieser Punkte ist ein einzelnes Atom; wenn ein Atom in der Molekel wenigstens zweimal so schwer ist als eins der anderen, so verursacht nur das von diesen Atomen allein gebildete Gitter die Beugungsmuster. Zwei Atome von fast demselben Atomgewicht sind als Beugungs. mittelpunkte fast gleichwertig. Die leichteren Atome der Molekel sind nicht eng um das schwere Atom, das das Beugungsraumgitter bildet, in der Runde angeordnet, sondern nehmen mittlere Lagen ein. Z. B. hat im Natriumchlorid das Natriumatom sechs benachbarte Chloratome in gleicher Entfernung, mit denen es sich paaren kann zur Bildung einer Molekel $\mathrm{NaCl}$. Die Reflexionskurven und Beugungsmuster der Alkalihalogenide weisen übereimmend auf dieselbe Struktur für diese Salze, die Atome sind in einem einfachen kubischen Raummuster in solcher Weise angeordnet, daB Reihen parallel zu den kubischen Achsen abwechselnd Atome beider Art enthalten. Die Zuordnung eines einzigen schweren Atomes zu jedem Punkt des Raumgitters wird angedeutet durch die Tatsache, daB die Masse eines jeden Punktes proportional ist dem Molargewicht des Stoffes, wenn jedes Mol ein schweres Atom enthält. Diese Beziehung erhält man aus den Reflexionskurven verschiedener Kristalle. Die Kenntnis der Masse eines Wasserstoffatomes ermöglicht die Berechnung der wirklichen Dimensionen eines Kristallgitters, so daB man dann die Wellenlänge der homogenen Komponenten eines X-Strahlenbündels in Zentimetern finden kann; dies 
war der Zweck, mit dem diese Analysen der Kristallstruktur aufgenommen wurden.

Zum SchluB möchte ich Herrn Professor Pope für seine freundliche Unterstützung und sein Interesse meinen Dank aussprechen. Dr. Hutschinson hat mit groBer Freundlichkeit die einzigen vorhandenen Experimentalschwierigkeiten überwinden helfen, indem er die nötigen Kristallschnitte zur Verfügung stellte. Ohne seine Hilfe wäre es unmöglich gewesen, die sehr groBe Zahl der hier benutzten Photographien zu erhalten. Diese Photographien sind hergestellt worden im Cavendish-Laboratorium, und ich möchte Professor Sir J.J. Tromson für sein freundliches Interesse an diesen Versuchen danken. Die Messungen mit dem X-Strahlenspektroskop sind, wie bereits angegeben, von meinem Vater im Laboratorium der Universität zu Leeds ausgeführt.

Cambridge (England) University, Trinity College.

Bei der Redaktion eingegangen am 14. Juli 1914. 